\title{
Phenomenology of the inert doublet model with a global $U(1)$ symmetry
}

\author{
Adil Jueid, ${ }^{1, *}$ Jinheung Kim $\odot,{ }^{1, \dagger}$ Soojin Lee $\odot,{ }^{1, \star}$ So Young Shim $\odot,{ }^{2, \S}$ and Jeonghyeon Song $\oplus^{1, \|}$ \\ ${ }^{1}$ Department of Physics, Konkuk University, Seoul 05029, Korea \\ ${ }^{2}$ Center for Theoretical Physics of the Universe, Institute for Basic Science (IBS), Daejeon, 34126, Korea
}

(Received 22 June 2020; accepted 22 September 2020; published 9 October 2020)

\begin{abstract}
The inert doublet model is a minimal dark matter model with strong theoretical motivations, where the stability of dark matter is usually achieved by imposing a $Z_{2}$ parity. We promote the $Z_{2}$ parity into a global $U(1)$ symmetry and study its phenomenological implications. There are two characteristic features of the model: both the $C P$-even and $C P$-odd neutral inert scalars, $h_{1}$ and $h_{2}$, become DM candidates; the number of model parameters is one less than that with $Z_{2}$ parity. We first analyze the constraints from LEP experiments, electroweak precision tests, theoretical stability, Higgs precision data, dark matter relic density, and direct detection experiments. It is found that if the model is required to explain at least $10 \%$ of the observed relic density, the theory is extremely limited such that the dark matter mass is about $70 \mathrm{GeV}$ and the charged Higgs boson is not very heavy. Focusing on this narrow parameter space, we calculate the production cross sections of almost all the possible mono- $X$ and mono- $X X^{\prime}$ processes at the LHC. The mono- $W \gamma$ process is shown to have high discovery potential with the help of the decay of the intermediatemass charged Higgs boson into $W^{ \pm} h_{1,2}$. A search strategy is designed to increase the potential discovery of the model for the mono- $W \gamma$ signal at both the HL-LHC and the FCC-hh. The optimal cut on $E_{T}^{\text {miss }} / \sqrt{H_{T}}$ is suggested to maximize the signal significance, being about 0.76 at the HL-LHC and about 7.5 at the FCC-hh.
\end{abstract}

DOI: 10.1103/PhysRevD.102.075011

\section{INTRODUCTION}

One of the most convincing pieces of evidence that the standard model (SM) is not the final theory of particle physics is the observed dark matter (DM) in the Universe [1]. Not knowing what it is yet, DM searches have been enthusiastically performed in three directions, direct detection, indirect detection, and its production at high energy colliders. Undeniably, a theory is essential in understanding all the different experimental results as a whole. A convenient approach to the theory of DM is through high-dimensional operators in the effective field theory [2-9] or through simplified DM models [10-20]. Nevertheless, studying one complete DM model has enormous advantages, especially for the DM searches at a high energy collider. For example, if the theory accommodates other new heavy particles decaying into a DM

\footnotetext{
*adil.hep@gmail.com

jinheung.kim1216@gmail.com

*soojinlee957@gmail.com

\$soyoung@ibs.re.kr

"jhsong@konkuk.ac.kr
}

Published by the American Physical Society under the terms of the Creative Commons Attribution 4.0 International license. Further distribution of this work must maintain attribution to the author(s) and the published article's title, journal citation, and DOI. Funded by SCOAP ${ }^{3}$. particle and an SM particle, some mono- $X X^{\prime}$ processes can be as important as mono- $X$ processes. We may have a different golden mode. In addition, a complete theory makes it possible to require theoretical stability and the compatibility with the electroweak precision data. The comprehensive study of the whole constraints including the DM observations shall significantly limit the viable region of the parameter space of the model. When the allowed parameter space is narrow enough, we could predict more definite signatures at a high energy collider. The inevitable weakness, the model-dependence, is something we can overcome only by dedicated studies on the phenomenology of each viable DM model.

One good example of theoretically well-motivated DM models with a relatively small number of parameters is the inert doublet model (IDM) with $Z_{2}$ parity [21]. As one of the simplest extensions of the SM, the IDM introduces an additional Higgs doublet field $\Phi^{\prime}$ which is odd under the $Z_{2}$ parity transformation. The model has drawn a lot of interest due to its capabilities such as triggering the first order electroweak phase transition [22-25], and generating neutrino masses [26]. Most of all, $\Phi^{\prime}$ that has neither a vacuum expectation value nor couplings to the SM fermions provides good DM candidates, neutral inert scalar bosons [27-31]. In the literature, various phenomenological implications of the model have been extensively studied [32-59]. 
Further simplification of the IDM was made by introducing a Peccei-Quinn symmetry to protect the mass degeneracy between the lightest and next-to-lightest neutral scalars for the inelastic DM-nucleus scattering [60-64]. Another kind of its extension introduced a minimal hidden sector with $U(1)_{\mathrm{CW}}$ gauge symmetry and a complex scalar [65]. Recently, the idea was extended to explore the mass degeneracies among some of the scalar bosons in various multi Higgs doublet models [66]. Focusing on the IDM, we find that promoting the $Z_{2}$ parity into a global $U(1)$ symmetry has two immediate consequences: (i) two neutral inert scalar bosons become DM particles; (ii) the number of model parameters is one less than that of the IDM with $Z_{2}$ parity. We naturally expect that the parameter space will be very strongly restricted by theoretical and experimental constraints, which should be investigated by a comprehensive study on the phenomenology of the model. Thus, our first purpose in this paper is to perform a comprehensive analysis of this model. With the result of the allowed (possibly very small) parameter space, we can assess all possible mono- $X$ and mono- $X X^{\prime}$ processes at the highluminosity LHC (HL-LHC) at $\sqrt{s}=14 \mathrm{TeV}$ with $3 \mathrm{ab}^{-1}$ [67] and the FCC-hh at $\sqrt{s}=100 \mathrm{TeV}$ and $30 \mathrm{ab}^{-1}$ [68], and suggest a golden mode for this model.

The paper is organized in the following way. In Sec. II, we briefly review the IDM with a global $U(1)$ symmetry. Section III deals with various constraints such as LEP experiments, the electroweak oblique parameters, the stability of scalar potential, unitarity, Higgs precision data including the Higgs invisible decay rate and $\kappa_{\gamma}$, DM relic density, and direct detection experiments. In Sec. IV, we calculate the total production cross sections of major mono$X$ and mono- $X X^{\prime}$ processes at the HL-LHC. Projecting the current direct DM searches onto the HL-LHC, we will suggest that the mono- $W \gamma$ is one of the most efficient channels to probe the model. In Sec. V, we present a search strategy to look for the model in the mono- $W \gamma$ process at the HL-LHC and FCC-hh. We conclude in Sec. VI.

\section{BRIEF REVIEW OF THE IDM WITH A CONTINUOUS $U(1)$ SYMMETRY}

In the IDM, the scalar sector is augmented by one extra $S U(2)_{L}$ doublet $\Phi^{\prime}$, in addition to the SM one $\Phi$. And we introduce a global $U(1)$ symmetry, under which $\Phi$ and $\Phi^{\prime}$ transform as

$$
\Phi \rightarrow \Phi, \quad \Phi^{\prime} \rightarrow e^{i \theta} \Phi^{\prime} .
$$

The fact that the extra doublet $\Phi^{\prime}$ has a nonzero $U(1)$ charge implies that its vacuum expectation value is vanishing. We have

$$
\left\langle\Phi_{0}\right\rangle=\frac{v}{\sqrt{2}} \approx 174 \mathrm{GeV}, \quad\left\langle\Phi_{0}^{\prime}\right\rangle \equiv \frac{v_{D}}{\sqrt{2}}=0 .
$$

In the unitary gauge, $\Phi$ and $\Phi^{\prime}$ are written as

$$
\Phi=\left(\begin{array}{c}
0 \\
\frac{1}{\sqrt{2}}(v+H)
\end{array}\right), \quad \Phi^{\prime}=\left(\begin{array}{c}
H^{+} \\
\frac{1}{\sqrt{2}}\left(h_{1}+i h_{2}\right)
\end{array}\right) .
$$

The particle spectrum of the model consists of five scalar states, the SM Higgs boson $H$ and the inert scalar bosons $h_{1}, h_{2}$, and $H^{ \pm}$. Although $h_{1}$ and $h_{2}$ have opposite $C P$ parities, we cannot tell which one is which, because the $C P$ transformation properties of $h_{1}$ and $h_{2}$ are exchanged under rephasing of $\Phi^{\prime} \rightarrow i \Phi^{\prime}$ [50]. The most general renormalizable and $C P$ invariant scalar potential that preserves the additional $U(1)$ symmetry is given by

$$
\begin{aligned}
V\left(\Phi, \Phi^{\prime}\right)= & -\mu_{1}^{2} \Phi^{\dagger} \Phi+\mu_{2}^{2} \Phi^{\prime \dagger} \Phi^{\prime}+\lambda_{1}\left(\Phi^{\dagger} \Phi\right)^{2}+\lambda_{2}\left(\Phi^{\prime \dagger} \Phi^{\prime}\right)^{2} \\
& +\lambda_{3}\left(\Phi^{\dagger} \Phi\right)\left(\Phi^{\prime \dagger} \Phi^{\prime}\right)+\lambda_{4}\left(\Phi^{\dagger} \Phi^{\prime}\right)\left(\Phi^{\prime \dagger} \Phi\right),
\end{aligned}
$$

where $m_{1}^{2}>0$ and $\mu_{2}^{2}>0$. The usual $\lambda_{5}$ term, proportional to $\left\{\left(\Phi^{\dagger} \Phi^{\prime}\right)^{2}+\right.$ H.c. $\}$, is prohibited by the $U(1)$ symmetry. Therefore, the absence of this term leads to the mass degeneracy between $h_{1}$ and $h_{2}$ :

$$
M_{h_{1}}=M_{h_{2}} \equiv M_{S}
$$

Note that this mass degeneracy persists at all orders in perturbation theory since it is protected by the global $U(1)$ symmetry. In what follows, we will call this model the IDM- $U(1)$ to distinguish it from the ordinary IDM model with $Z_{2}$ parity.

The IDM- $U(1)$ contains six additional parameters, $\mu_{1}^{2}$, $\mu_{2}^{2}$, and $\lambda_{1,2,3,4}$. Since the Higgs boson mass fixes two parameters $\mu_{1}^{2}$ and $\lambda_{1}$ as

$$
\mu_{1}^{2}=\frac{m_{H}^{2}}{2}, \quad \lambda_{1}=\frac{m_{H}^{2}}{2 v^{2}},
$$

we are left with only four extra parameters. We take the physical parameter basis defined by

$$
\left\{M_{S}, M_{H^{ \pm}}, \lambda_{L}, \lambda_{2}\right\},
$$

where $\lambda_{L}=\left(\lambda_{3}+\lambda_{4}\right) / 2$. The other model parameters are obtained from the following relations:

$$
\begin{aligned}
& \mu_{2}^{2}=M_{S}^{2}-\lambda_{L} v^{2}, \\
& \lambda_{3}=2\left[\lambda_{L}+\frac{M_{H^{ \pm}}^{2}-M_{S}^{2}}{v^{2}}\right], \\
& \lambda_{4}=-\frac{2}{v^{2}}\left(M_{H^{ \pm}}^{2}-M_{S}^{2}\right) .
\end{aligned}
$$

The interaction Lagrangian of the SM Higgs boson $H$ with the SM particles is the same as in the SM. The gauge interaction Lagrangian of the inert scalar bosons is 


$$
\begin{aligned}
\mathcal{L}_{\text {gauge }}= & \frac{1}{2} g_{Z} Z^{\mu} h_{2} \stackrel{\leftrightarrow}{\partial}_{\mu} h_{1}-\frac{1}{2} g\left[i W_{\mu}^{+} H^{-} \stackrel{\leftrightarrow}{\partial}_{\mu}\left(h_{1}+i h_{2}\right)+\text { H.c. }\right] \\
& +i\left[e A^{\mu}+g_{H^{ \pm}} Z^{\mu}\right] H^{+} \stackrel{\leftrightarrow}{\partial}_{\mu} H^{-}+\left(\frac{1}{4} g^{2} W_{\mu}^{+} W^{-\mu}+\frac{1}{8} g_{Z}^{2} Z_{\mu} Z^{\mu}\right)\left(h_{1}^{2}+h_{2}^{2}\right) \\
& +\left[\frac{1}{2} g^{2} W_{\mu}^{+} W^{-\mu}+e^{2} A_{\mu} A^{\mu}+g_{H^{ \pm}}^{2} Z_{\mu} Z^{\mu}+2 e g_{H^{ \pm}} A_{\mu} Z^{\mu}\right] H^{+} H^{-} \\
& +\left[\left(\frac{1}{2} e g A^{\mu} W_{\mu}^{+}-\frac{1}{2} g_{Z} g s_{W}^{2} Z^{\mu} W_{\mu}^{+}\right) H^{-}\left(h_{1}+i h_{2}\right)+\text { H.c. }\right],
\end{aligned}
$$

where $g_{H^{ \pm}}=g_{Z}\left(1 / 2-s_{W}^{2}\right), \quad g_{Z}=g / c_{W}, \quad s_{W}=\sin \theta_{W}$, $c_{W}=\cos \theta_{W}$, and $\theta_{W}$ is the electroweak mixing angle. The interactions of the inert scalar bosons to a single $H$ are described by

$$
\mathcal{L}_{\text {scalar }} \supset-v H\left[\lambda_{L}\left(h_{1}^{2}+h_{2}^{2}\right)+\lambda_{3} H^{+} H^{-}\right] .
$$

Since the SM fields do not have the $U(1)$ charge, the Yukawa couplings of the inert scalar bosons to the SM fermions vanish. Consequently, the decays of the new scalar bosons are very simple. Both $h_{1}$ and $h_{2}$, as the lightest particles with nonzero $U(1)$ charge, do not decay and become the DM candidates. The charged Higgs boson $H^{ \pm}$exclusively decays into $W^{ \pm(*)} h_{1,2}$ :

$$
\mathcal{B}\left(H^{ \pm} \rightarrow W^{ \pm(*)} h_{1,2}\right)=1
$$

The final comment is that the $U(1)$ symmetry, which protects the mass degeneracy $M_{h_{1}}=M_{h_{2}}$, should be softly broken to avoid large inelastic DM-nucleus scattering in the direct detection experiments, through $Z-h_{1}-h_{2}$ vertex $[64,69]$. If we allow very small mass difference like $\delta m\left(\equiv M_{h_{2}}-M_{h_{1}}\right) \simeq 200 \mathrm{keV}$, however, the inelastic scattering does not occur because of the kinematical threshold for the DM to scatter inelastically off a nucleus. We attribute the soft-breaking of the $U(1)$ symmetry to some higher dimensional operators originated from the unknown UV theory [62]. Note that this small mass splitting implies $\lambda_{5} \sim$ $\mathcal{O}\left(10^{-8}\right)$ so the collider phenomenology, as well as all the constraints to be discussed in the next section, is practically the same as in the IDM with exact $U(1)$ symmetry.

\section{THEORETICAL AND EXPERIMENTAL CONSTRAINTS}

Compared to the IDM with discrete $Z_{2}$ parity, the IDM$U(1)$ is severely restricted due to the compressed spectrum in the neutral component of the scalar doublet $\Phi^{\prime}$. In this section, we consider the following constraints:

(i) Z-decay width and the bounds from the searches of charginos at LEP;

(ii) The electroweak precision data (EWPD) encoded in the Peskin-Takeuchi oblique parameters; (iii) Theoretical constraints from boundness-from-below (BFB) of the scalar potential, perturbativity, and unitarity;

(iv) Searches of Higgs boson invisible decays at the LHC and the precision measurement of the Higgs coupling modifier to a photon pair, $\kappa_{\gamma}$;

(v) DM relic density and the bounds from DM direct detection experiments.

The bounds from the direct search of a pseudo-scalar boson $A$ reported by the LEP, Tevatron, and LHC are not relevant in this model since all of these searches depend on the fermionic decay modes of $A$ while, in our model, the pseudoscalar state (either $h_{1}$ or $h_{2}$ ) does not decay.

\section{A. LEP experiments and electroweak precision data}

In the IDM- $U(1)$, the $Z$ boson decays into $h_{1} h_{2}$ if kinematically allowed: see Eq. (9). The precise measurement of the total width of the $Z$ boson excludes $M_{S}<$ $m_{Z} / 2$ [70]. In addition, the reinterpretation of the chargino pair production as $e^{+} e^{-} \rightarrow H^{+} H^{-}[71,72]$ puts a lower bound on the charged Higgs boson mass. In summary, the null results from LEP require

$$
M_{S}>\frac{m_{Z}}{2}, \quad M_{H^{ \pm}}>70 \mathrm{GeV} .
$$

One of the most significant constraints on the IDM- $U(1)$ is from the EWPD oblique parameters, $S$ and $T$. The contributions of the inert scalar bosons to the $S$ and $T$ parameters can be written as

$$
\begin{aligned}
& S=\frac{1}{12 \pi} \ln \frac{M_{S}^{2}}{M_{H^{ \pm}}^{2}}, \\
& T=\frac{1}{16 \pi^{2} \alpha v^{2}} F\left(M_{H^{ \pm}}^{2}, M_{S}^{2}\right),
\end{aligned}
$$

where the loop function $F(x, y)$ is

$$
F(x, y)= \begin{cases}\frac{x+y}{2}-\frac{x y}{x-y} \ln \frac{x}{y}, & \text { if } x \neq y \\ 0, & \text { if } x=y .\end{cases}
$$

The current best-fit results are given by [70]

$S=0.02 \pm 0.07, \quad T=0.07 \pm 0.06, \quad \rho_{S T}=0.92$ 


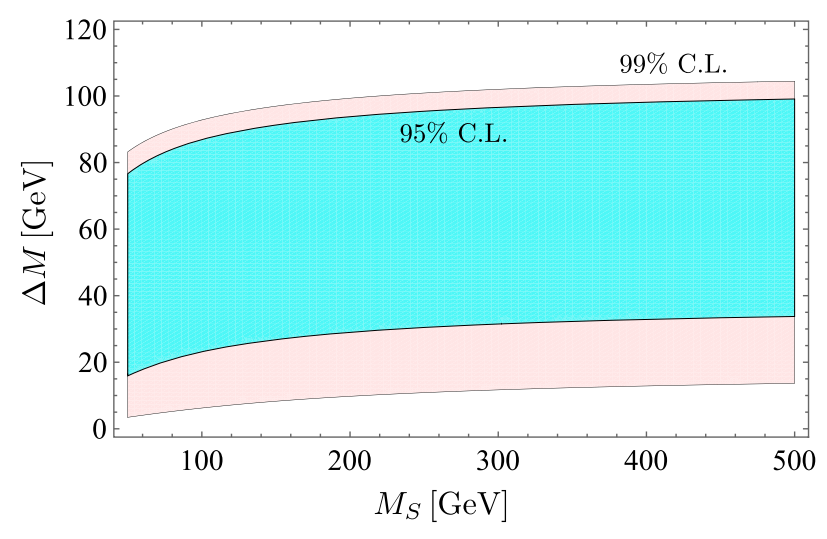

FIG. 1. Allowed region of $\left(M_{S}, \Delta M\right)$ by the electroweak oblique parameters $S$ and $T$ at $95 \%$ and $99 \%$ C.L., where $M_{S} \equiv$ $M_{h_{1}}=M_{h_{2}}$ and $\Delta M \equiv M_{H^{ \pm}}-M_{S}$. The strong correlation between $S$ and $T, \rho_{S T}=0.92$, is included in the $\chi^{2}$ calculation.

where $U=0$ is assumed, and with $\rho_{S T}$ is the correlation between $S$ and $T$. In order to obtain the allowed region in the mass spectrum of the model, we minimize the following $\chi^{2}$ :

$\chi^{2}=\sum_{\mathcal{O}=S, T} \frac{\left(\mathcal{O}-\mathcal{O}_{\exp }\right)^{2}}{\sigma_{\mathcal{O}}^{2}\left(1-\rho_{S T}\right)}-2 \rho_{S T} \frac{\left(S-S_{\exp }\right)\left(T-T_{\exp }\right)}{\sigma_{S} \sigma_{T}\left(1-\rho_{S T}\right)}$,

where $\sigma_{\mathcal{O}}$ is the error on the observable $\mathcal{O}$.

In Fig. 1, we present the allowed region of $\left(M_{S}, \Delta M\right)$ by the electroweak oblique parameters $S$ and $T$ at $95 \%$ and 99\% C.L., where $\Delta M \equiv M_{H^{ \pm}}-M_{S}$. We can see that the constraints from EWPD on the mass spectrum are very stringent. In fact, the mass difference between the charged Higgs boson and the DM cannot be too large or too small, $10 \lesssim \Delta M \lesssim 100 \mathrm{GeV}$. The upper bound on $\Delta M$ implies that we cannot arbitrarily ignore $H^{ \pm}$by decoupling it from the model. On the other hand, the presence of the lower bound on $\Delta M$ excludes the possibility of the total mass degeneracy, $M_{h_{1}}=M_{h_{2}} \simeq M_{H^{ \pm}} .{ }^{1}$ Note that the correlation $\rho_{S T}$ plays a crucial role here: the allowed range would be $0 \leq \Delta M \lesssim$ $190 \mathrm{GeV}$ if we assumed that $S$ and $T$ are uncorrelated.

\section{B. Constraints from the theoretical stability and the Higgs precision data}

The parameters of the scalar potential should satisfy the following conditions for the feasibility of the model.
(1) Perturbativity:

$$
\left|\lambda_{1,2,3,4}\right| \leq 8 \pi .
$$

(2) BFB condition for the scalar potential:

$$
\lambda_{2}>0, \quad \lambda_{3}>-2 \sqrt{\lambda_{1} \lambda_{2}}, \quad \lambda_{L}>-\sqrt{\lambda_{1} \lambda_{2}} .
$$

(3) Electroneutrality of the vacuum and the DM particle:

$$
\lambda_{4}<0
$$

(4) Tree level unitarity: the eigenvalues of the $S$ matrix for the scalar-scalar scattering processes should satisfy [40]

$$
\left|a_{i}\right| \leq 8 \pi, \quad(i=1,2, \ldots, 10)
$$

where

$$
\begin{aligned}
& a_{1,2}=\lambda_{3} \pm \lambda_{4}, \quad a_{3}=\lambda_{3}, \quad a_{4}=\lambda_{3}+2 \lambda_{4}, \\
& a_{5,6}=-\lambda_{1}-\lambda_{2} \pm \sqrt{\left(\lambda_{1}-\lambda_{2}\right)^{2}+\lambda_{4}^{2}}, \\
& a_{7,8}=-3 \lambda_{1}-3 \lambda_{2} \pm \sqrt{9\left(\lambda_{1}-\lambda_{2}\right)^{2}+\left(2 \lambda_{3}+\lambda_{4}\right)^{2}}, \\
& a_{9,10}=-\lambda_{1}-\lambda_{2} \pm\left|\lambda_{1}-\lambda_{2}\right| .
\end{aligned}
$$

(5) The invisible decay of the Higgs boson. If $M_{S}<m_{H} / 2$, the SM-like Higgs boson decays invisibly via $H \rightarrow h_{1} h_{1} / h_{2} h_{2}$. The upper bound on the branching ratio of the invisible Higgs decay constrains $\lambda_{L}$ as

$$
\left|\lambda_{L}\right|<\left[\frac{\pi g^{2} m_{H} \Gamma_{H}^{\mathrm{SM}}}{\beta_{S} m_{W}^{2}\left(\frac{1}{\mathcal{B}_{\text {inv }}^{\max }}-1\right)}\right]^{1 / 2},
$$

where $\beta_{S}=\sqrt{1-4 M_{S}^{2} / m_{H}^{2}}$. In our model with two DM particles, the upper bound on $\left|\lambda_{L}\right|$ is smaller by a factor of $1 / \sqrt{2}$ than that with one DM particle [50]. We adopt the latest ATLAS Higgs combined results [75], $\mathcal{B}_{\text {inv }}<0.30$ at $95 \%$ C.L.

(6) The diphoton decay rate of the Higgs boson, which is modified by the contributions from the charged Higgs boson. The Higgs coupling modifier $\kappa_{\gamma}$ in the $\operatorname{IDM}-U(1)$ is [40-42]

$$
\kappa_{\gamma}=\left|\frac{\sum_{i=t, b, \tau} N_{i}^{C} Q_{i}^{2} A_{1 / 2}\left(\tau_{f}^{h}\right)+A_{1}\left(\tau_{W}^{h}\right)+\frac{\lambda_{3} v^{2}}{2 M_{H^{ \pm}}^{2}} A_{0}\left(\tau_{H^{ \pm}}^{h}\right)}{\sum_{i=t, b, \tau} N_{i}^{C} Q_{i}^{2} A_{1 / 2}\left(\tau_{f}^{h}\right)+A_{1}\left(\tau_{W}^{h}\right)}\right|,
$$

\footnotetext{
${ }^{1}$ If $\Delta M \simeq 0.2 \mathrm{GeV}$ [73], we could observe the disappearing charged track signatures at the LHC from the $H^{ \pm}$production and its subsequent soft decays [74].
} 
where $N_{i}^{C}$ is the color factor of the fermion, $\tau_{i}^{h}=m_{H}^{2} /\left(4 m_{i}^{2}\right)$, and loop functions $A_{0,1 / 2,1}(\tau)$ can be found in, e.g., [42]. We take $\left|\kappa_{\gamma}\right|=1.05 \pm$ 0.09 [75].

Before presenting the results of the constraints, we show that the BFB condition makes the inert vacuum in Eq. (2) be the only true vacuum in the IDM-U(1). The scalar potential in Eq. (4) has an additional minimum, called the mixed extremum [76], having the vacuum expectation value of $\Phi^{\prime}$ as

$$
\left(v_{D}^{\text {mix }}\right)^{2}=-\frac{\lambda_{1} \mu_{2}^{2}+\lambda_{L} m_{1}^{2}}{\lambda_{1} \lambda_{2}\left(1-R^{2}\right)}
$$

where $R \equiv \lambda_{L} / \sqrt{\lambda_{1} \lambda_{2}}$. The energy difference between the inert and mixed vacua is

$$
\mathcal{E}_{\text {inert }}-\mathcal{E}_{\text {mixed }}=\frac{\left(\lambda_{L} m_{1}^{2}+\lambda_{1} \mu_{2}^{2}\right)^{2}}{4 \lambda_{1}^{2} \lambda_{2}\left(1-R^{2}\right)} .
$$

If $\mathcal{E}_{\text {inert }}-\mathcal{E}_{\text {mixed }}<0$, the inert vacuum becomes the global minimum. The BFB condition in Eq. (18) leads to $R>-1$. When $1<R^{2}, \mathcal{E}_{\text {inert }}<\mathcal{E}_{\text {mixed }}$ is automatically satisfied. If $R^{2}<1,\left(v_{D}^{\text {mix }}\right)^{2}$ with Eqs. (6) and (8) becomes

$$
\left(v_{D}^{\operatorname{mix}}\right)^{2}=-\frac{m_{H}^{2}}{2 v^{2}} \frac{M_{S}^{2}}{\lambda_{1} \lambda_{2}\left(1-R^{2}\right)}<0
$$

which disqualifies the mixed vacuum as a vacuum solution. In summary, the IDM- $U(1)$ accommodates only one true vacuum, the inert vacuum.

In Fig. 2, we present the excluded regions of $\left(M_{S}, \lambda_{L}\right)$ by the unitarity, the BFB scalar potential, the Higgs coupling modifier $\kappa_{\gamma}$, and the Higgs invisible decay rate. The perturbativity imposes weaker constraints than the unitarity

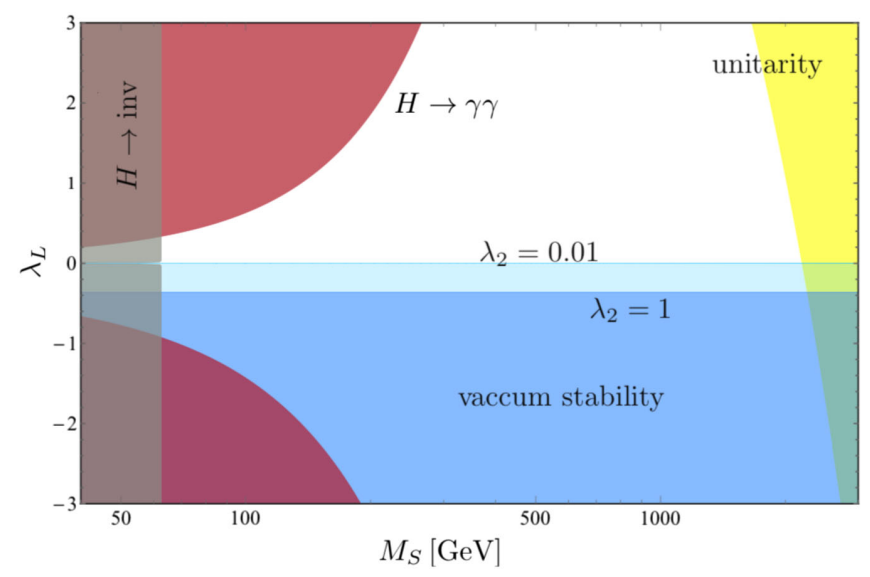

FIG. 2. Excluded region of $\left(M_{S}, \lambda_{L}\right)$ by the unitarity, the bounded-from-below scalar potential, the diphoton decay rate of the Higgs boson, and the Higgs invisible decay rate. We take $M_{H^{ \pm}}=M_{S}+85 \mathrm{GeV}$ for two cases of $\lambda_{2}=0.01$ and $\lambda_{2}=1$. which do not appear in the figure. We take $\Delta M=85 \mathrm{GeV}$ for two cases of $\lambda_{2}=0.01$ and $\lambda_{2}=1$. Note that the exclusions barely depend on the mass splitting $\Delta M$ : if we set $\Delta M=40 \mathrm{GeV}$, nothing will practically change. The unitarity condition excludes heavy DM masses, $M_{S}$, since the scalar quartic couplings $\lambda_{3}$ and $\lambda_{4}$ are proportional to $M_{S}$ for a given $\Delta M$ : see Eq. (8). The $\kappa_{\gamma}$ excludes the region with $M_{H^{ \pm}} \lesssim 200 \mathrm{GeV}$ and sizable $\lambda_{L}$. The asymmetry of the excluded region by $\kappa_{\gamma}$ about $\lambda_{L}=0$ is attributed to the destructive (constructive) interference between $H^{ \pm}$and $W^{ \pm}$ contributions for $\lambda_{3}>0\left(\lambda_{3}<0\right)$ [48,77]. The Higgs invisible decay rate limits the value of $\lambda_{L}$ very strongly in the mass range of $M_{S}<m_{H} / 2$. For $M_{S}=60 \mathrm{GeV}$, the maximum allowed value of $\left|\lambda_{L}\right|$ is only about 0.013 , which is too small to be seen in this linear scale figure.

\section{DM relic density and direct DM detection}

The DM relic density has been measured with high precision by the PLANCK experiment [78]:

$$
\Omega_{\mathrm{DM}}^{\text {Planck }} h^{2}=0.1184 \pm 0.0012 .
$$

In the IDM-U(1), both $h_{1}$ and $h_{2}$ contribute to the relic density. With the possibility that there exist other sources of DM, we avoid the DM overabundance. At the same time, we do not allow too small contribution of our model. If the relic density of $h_{1}$ and $h_{2}$ cannot reach just $1 \%$ of the observed relic density, there must be a more important new physics model providing DM candidates: the motivation for studying the phenomenology of the IDM- $U(1)$ is getting very weak. Therefore we demand

$$
0.01<\frac{\Omega_{h_{1,2}}}{\Omega_{\mathrm{DM}}^{\text {Plank }}}<1 .
$$

We also consider the constraints of direct detection experiments. To do so, we calculate the spin-independent DM-nucleon elastic scattering cross section $\left(\sigma_{\mathrm{SI}}\right)$ by using the micrOMEGAs package [79]. We then require that $\sigma_{\mathrm{SI}}$ is below the bounds reported on by the XENON1T experiment [80]. In cases that the relic density in our model is smaller than the Planck measurement, we use the rescaled cross section

$$
\hat{\sigma}_{\mathrm{SI}}=\frac{\Omega_{h_{1,2}} h^{2}}{\Omega_{\mathrm{DM}}^{\text {Planck }} h^{2}} \sigma_{\mathrm{SI}} .
$$

Figure 3 shows the allowed region by the relic density and the XENON1T experiment. The pink region is allowed by the condition of $0.01<\Omega_{h_{1,2}} / \Omega_{\mathrm{DM}}^{\text {Planck }}<1$, permitting a wide mass range of the inert DM particles as long as $\left|\lambda_{L}\right|$ is small enough. The maximum of $\Omega_{h_{1,2}} / \Omega_{\mathrm{DM}}^{\text {Planck }}$ is only $\sim 25 \%$, occurring at $M_{S} \simeq 85 \mathrm{GeV}$ and $\lambda_{L} \simeq-0.13$. The inert dark scalars alone cannot explain the observed $\Omega_{\mathrm{DM}}^{\text {Planck }}$. 


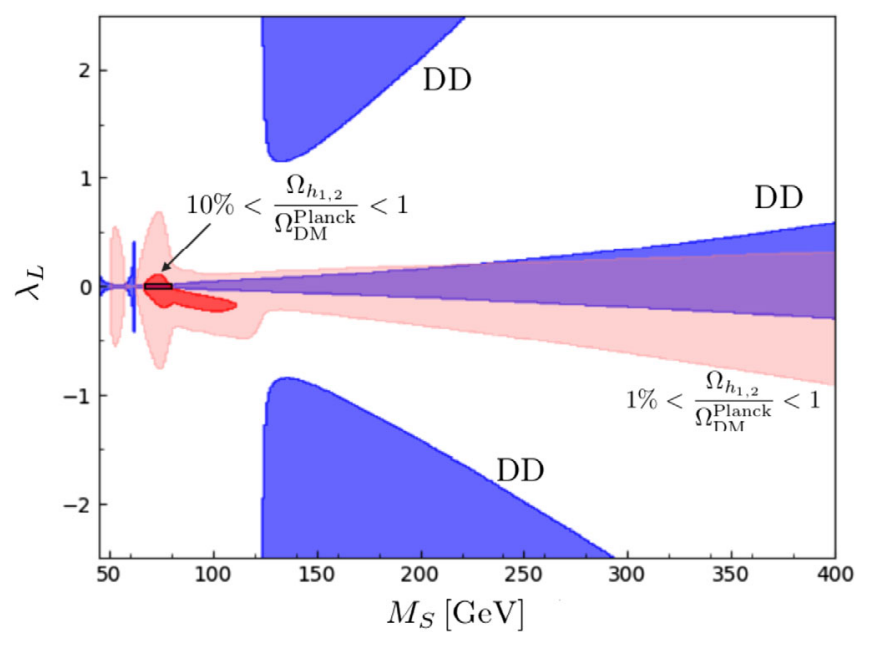

FIG. 3. Allowed region of $\left(M_{S}, \lambda_{L}\right)$ by the relic density and the direct detection of the DM, by demanding $0.01<\Omega_{h_{1}, h_{2}} /$ $\Omega_{\mathrm{DM}}^{\text {Planck }}<1$ (pink region), $0.1<\Omega_{h_{1}, h_{2}} / \Omega_{\mathrm{DM}}^{\text {Planck }}<1$ (red region), and the XENON1T experiment (blue region). We set $M_{H^{ \pm}}=M_{S}+85 \mathrm{GeV}$.

If we demand $\Omega_{h_{1,2}} / \Omega_{\mathrm{DM}}^{\text {Planck }}>10 \%$ (red region), only a small portion of the parameter space around $65 \lesssim M_{S} \lesssim$ $115 \mathrm{GeV}$ and $\left|\lambda_{L}\right| \ll 1$ survives. The blue regions are allowed by the XENON1T experiment, consisting of the horizontal region with small $\left|\lambda_{L}\right|$ and two triangular regions with $\left|\lambda_{L}\right|>1$. The triangular regions are permitted, because of the suppression from very small $\Omega_{h_{1,2}} / \Omega_{\mathrm{DM}}^{\text {Planck }}$ in Eq. (29). The overlapping region is allowed by the combination of the two constraints. If we demand $\Omega_{h_{1,2}} / \Omega_{\mathrm{DM}}^{\text {Planck }}>10 \%$, the combined DM constraints exclude most of the parameter space, except for very narrow area (red region enclosed by black solid line) with $65 \lesssim M_{S} \lesssim 80 \mathrm{GeV}$ and $\left|\lambda_{L}\right|<0.01$.

\section{PROBING THE IDM- $U(1)$ AT THE LHC}

\section{A. Production of the inert DM associated with gauge bosons at the LHC}

The phenomenology of the IDM- $U(1)$ at the LHC is simple since the model contains only two neutral scalars $\left(h_{1}\right.$ and $h_{2}$ ), which will play the role of missing energy, and the charged Higgs boson $H^{ \pm}$decaying into $W^{ \pm(*)} h_{1,2}$. The production of the inert scalar bosons is mainly via the gauge bosons since the SM Higgs boson plays a minor role. The two production channels mediated by the SM Higgs boson, $g g \rightarrow H \rightarrow h_{i} h_{i}$ and $g g \rightarrow H \rightarrow H^{+} H^{-}$, are suppressed: the vertices $H-h_{1}-h_{1}$ and $H-h_{2}-h_{2}$ are proportional to the very small $\left|\lambda_{L}\right| \lesssim \mathcal{O}(0.01)$; both channels are oneloop induced with the exchange of an off-shell SM Higgs boson. In summary, the production of inert scalars are, to a large extent, model-independent due to the sole contribution of gauge couplings.

For each production channel of the inert scalar bosons, we attach gauge bosons in order to tag the missing energy signal. ${ }^{2}$ Limiting up to two gauge bosons as tagging particles, the following schematic processes are feasible at the LHC:

(i) $\left[q \bar{q} \rightarrow Z^{*} \rightarrow h_{1} h_{2}\right] \oplus g / \gamma / W^{ \pm} / Z$;

(ii) $\left[q \bar{q}^{\prime} \rightarrow W^{ \pm *} \rightarrow H^{ \pm} h_{1,2} \rightarrow W^{ \pm(*)} h_{1,2} h_{1,2}\right] \oplus \gamma / Z / W^{ \pm}$;

(iii) $q q \rightarrow Z^{*} / \gamma^{*} \rightarrow H^{+} H^{-} \rightarrow W^{+} W^{-} h_{1,2} h_{1,2}$;

(iv) $q \bar{q} \rightarrow Z^{*} \rightarrow Z h_{i} h_{i} \quad$ and $\quad q \bar{q}^{\prime} \rightarrow W^{ \pm *} \rightarrow W^{ \pm} h_{i} h_{i}$ $(i=1,2)$;

(v) $g g \rightarrow H \rightarrow H^{+} H^{-} \rightarrow W^{+} W^{-} h_{1,2} h_{1,2}$.

In terms of final states, we have monojet, mono- $\gamma$, mono- $Z$, mono- $W$, mono- $W \gamma$, mono- $W Z$, and mono- $W W$ channels. As with the terminology of mono- $X$, the mono- $X X^{\prime}$ process means the production of $X X^{\prime}$ associated with large missing transverse energy.

Figure 4 presents the total production cross sections of various mono- $X$ and mono- $X X^{\prime}$ processes as a function of $M_{S}$ at the $14 \mathrm{TeV}$ LHC. We have used MadGraph5_aMC@NLO [81,82] with the IDM model file $[30,47]$ in the UFO format [83]. For $\lambda_{L}=0$ and $\lambda_{2}=0.5$, we consider two cases, $\Delta M=40 \mathrm{GeV}$ (left panel) and $\Delta M=85 \mathrm{GeV}$ (right panel). ${ }^{3}$ The mono-jet production cross section, which is independent of $\Delta M$, is the largest, about $500 \mathrm{fb}$ for $M_{S}=70 \mathrm{GeV}$. In the $\Delta M=$ $40 \mathrm{GeV}$ case, the production cross sections of mono- $V$ and mono- $V V^{\prime}$, where $V^{(\prime)}$ is an electroweak gauge boson, are very small. The mono- $V$ processes barely keep $\sigma_{\text {mono- } V} \sim$ $\mathcal{O}(1) \mathrm{fb}$ for $M_{S} \lesssim 130 \mathrm{GeV}$. The mono- $V V^{\prime}$ processes have much smaller cross sections, below $\sim 0.1 \mathrm{fb}$ even for $M_{S}=50 \mathrm{GeV}$.

In the $\Delta M=85 \mathrm{GeV}$ case, the production cross sections of the processes involving $W^{ \pm}$bosons are highly enhanced thanks to the on-shell decay of $H^{ \pm} \rightarrow W^{ \pm} h_{1,2}$. First, the mono- $W$ cross section becomes comparable to the monojet one for $M_{S} \lesssim 100 \mathrm{GeV}$ and larger for $M_{S} \gtrsim 100 \mathrm{GeV}$. ${ }^{4}$ The main production channel is $q \bar{q}^{\prime} \rightarrow$ $H^{ \pm}\left(\rightarrow W^{ \pm} h_{1,2}\right) h_{1,2}$, effectively a $2 \rightarrow 2$ process. The mono- $W W$ cross section is also enhanced, as the major production of $q \bar{q} \rightarrow H^{+}\left(W^{+} h_{1,2} h_{1,2}\right) H^{-}\left(\rightarrow W^{-} h_{1,2} h_{1,2}\right)$ is also a $2 \rightarrow 2$ process. The contribution from $g g \rightarrow H^{*} \rightarrow$ $\mathrm{H}^{+} \mathrm{H}^{-}$is minor, below 5\%. Finally, other interesting processes such as mono- $W \gamma$ and mono- $W Z$ yield quite sizable cross sections.

\footnotetext{
${ }^{2}$ There are other processes such as the mono-Higgs process and the vector boson fusion production of the inert scalar bosons. However, these processes are subleading and we ignore them in this work.

${ }^{3}$ The range of the scan over $M_{S}$ in this analysis is motivated by the fact that requiring sizable relic density prefers light DM mass. However, we note that our model can allow for heavy DM with mass above $400 \mathrm{GeV}$ : see, e.g., [28,84] for more details about the phenomenology of heavy DM in the IDM.

${ }^{4}$ The cross sections of the mono- $W$ process in the $\Delta M=$ $85 \mathrm{GeV}$ case are still consistent with the current ATLAS result of the search of DM in association with hadronically-decaying $W / Z$ channel [85].
} 

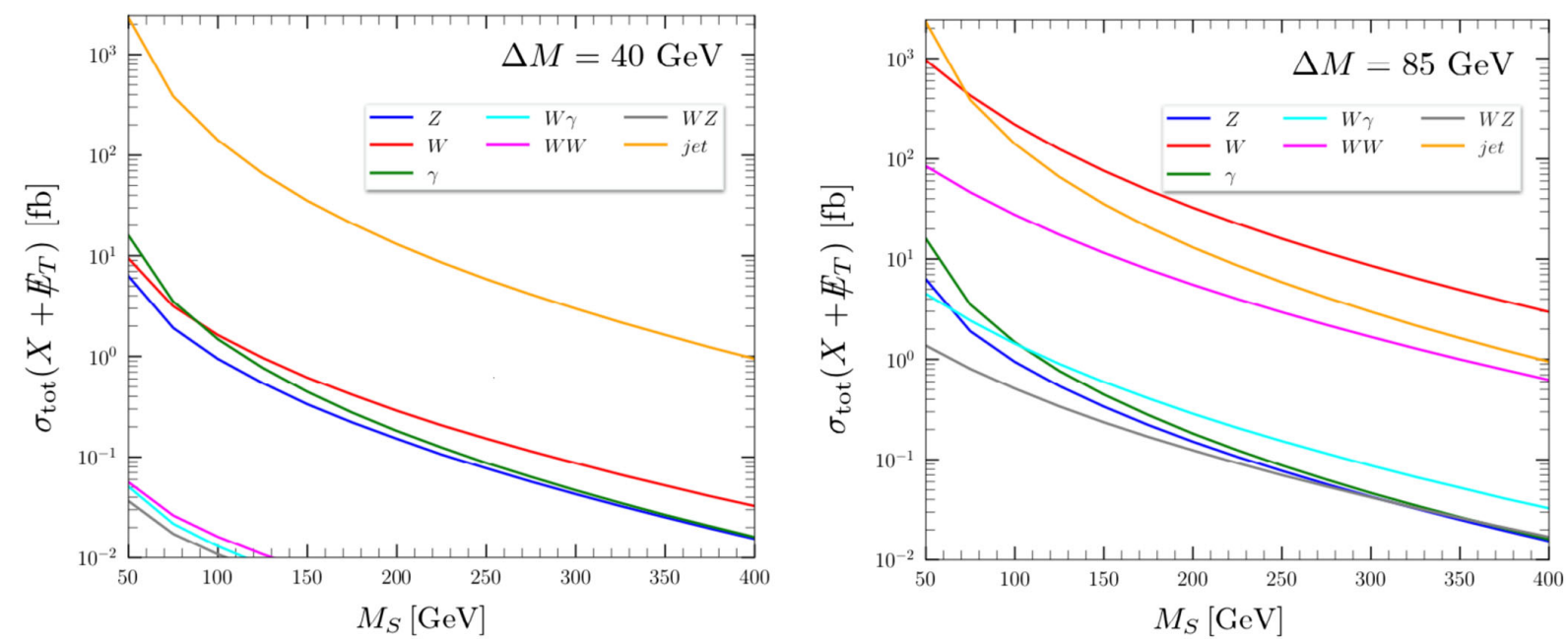

FIG. 4. The cross sections of mono- $X$ and mono- $X X^{\prime}$ processes at the $14 \mathrm{TeV}$ LHC as a function of $M_{S}$. We set $\lambda_{L}=0, \lambda_{2}=0.5$, $\Delta M=M_{H^{ \pm}}-M_{S}=40 \mathrm{GeV}$ (left panel), and $\Delta M=85 \mathrm{GeV}$ (right panel).

\section{B. Assessing the LHC sensitivities of various mono- $X$ and mono- $X X^{\prime}$ processes in probing the IDM- $U(1)$}

Before presenting our main results in the next section, we assess the LHC discovery prospects of some mono- $X$ and mono- $X X^{\prime}$ processes:

(i) Monojet process

In the IDM with $Z_{2}$ parity, the main production channel for the mono-jet process is $g g \rightarrow H^{*} \rightarrow$ $h_{1} h_{1} g$. In the IDM- $U(1)$, however, the vertex $H-$ $h_{i}-h_{i}(i=1,2)$ is very suppressed by the combined constraint from the relic density and the direct detection experiments (see Fig. 3). Therefore, the main contribution to the mono-jet signature is from $p p \rightarrow Z^{*} \rightarrow h_{1} h_{2} j$. In Ref. [54], this mode was studied as an exceptional case of the IDM- $Z_{2}$ by imposing the condition of $M_{h_{2}}-M_{h_{1}}=1 \mathrm{GeV}$ : it was concluded that this process does not reach the discovery at the HL-LHC for $M_{h_{1}} \gtrsim 50 \mathrm{GeV}$. It is difficult to probe the IDM- $U(1)$ through the monojet channel.

(ii) Mono- $W$ process

Even though the on-shell decay of $H^{ \pm}$into $W^{ \pm} \gamma$ helps to increase the total production cross section of the mono- $W$ process, about $0.5 \mathrm{pb}$ when $M_{S}=$ $70 \mathrm{GeV}$ and $\Delta M=85 \mathrm{GeV}$, it is not easy to probe this mode. For the hadronic decay of $W^{ \pm}$, the SM backgrounds such as $p p \rightarrow W^{ \pm} Z(\rightarrow \bar{\nu} \nu)$ and $p p \rightarrow$ $j j Z(\rightarrow \bar{\nu} \nu)$ are overwhelming. For the leptonic decay mode of $W^{ \pm}$, yielding the final state of $\ell+E_{T}^{\text {miss }}$, the irreducible SM background $p p \rightarrow$ $W^{ \pm}(\rightarrow \ell \nu)$ is enormous: the observed total cross section at $13 \mathrm{TeV}$ is $\sigma\left(p p \rightarrow W^{ \pm}\right) \simeq 97 \mathrm{nb}$ [86]. We found that both the signal and the background have very similar shapes in the main kinematic distributions such as the transverse momentum of the charged lepton, the missing transverse energy
$E_{T}^{\text {miss }}$, the total transverse hadronic energy $H_{T}$, and the imbalance between the charged lepton and the missing transverse energy $p_{T}^{\ell} / \vec{E}_{T}^{\text {miss }}$ [87]. The similarity is partially due to light DM mass. Considering other reducible backgrounds such as $t \bar{t}$, the Drell-Yan production of dilepton with one lepton escaping the detection, and the diboson $(W W, W Z$, and $Z Z$ ) productions, we expect that this mode is challenging to probe at the future LHC.

(iii) Mono- $Z$ and mono- $\gamma$ process

Both mono- $Z$ and mono- $\gamma$ processes have the production cross sections below $\sim 1 \mathrm{fb}$, which are almost independent of $\Delta M$. The cross sections are too small to probe the model. The current status of the DM searches through mono- $Z$ is $\sigma_{\text {mono- } Z} \lesssim 3 \mathrm{pb}$, at $\sqrt{s}=13 \mathrm{TeV}$ with the total integrated luminosity of $36.1 \mathrm{fb}^{-1}$ [85]. The projection to the HL-LHC is $\sigma_{\text {mono-Z }} \lesssim 300 \mathrm{fb}$. The IDM- $U(1)$ has no chance to be probed through the mono- $Z$ process. The mono- $\gamma$ process is also infeasible to probe in our model [88,89]. The main reason is that the $E_{T}^{\text {miss }}$ distribution of our signal, populated below $150 \mathrm{GeV}$, is very similar to the $\mathrm{SM}$ background.

(iv) Mono- $W W$ process

This process yields the clean dilepton plus missing transverse energy signal at the LHC [36,47]. According to the analysis in the IDM with $Z_{2}$ parity [36], the signal significance of this mode is extremely small to be $n_{s} / \sqrt{n_{b}} \simeq 0.02$, for $M_{H^{ \pm}}-M_{h_{1}}=50 \mathrm{GeV}$ and $M_{h_{2}}-M_{h_{1}}=10 \mathrm{GeV}$. Even though our model has a little larger cross section, it is not enough to enhance the signal-to-background ratio.

(v) Mono- $W \gamma$ process

When $\Delta M=85 \mathrm{GeV}$, the production cross section of mono- $W \gamma$ is about $2.8 \mathrm{fb}$ for $M_{S}=70 \mathrm{GeV}$. 
The ATLAS [90] and CMS [91] collaborations analyzed this mode in the search for supersymmetry with a general gauge-mediated mechanism. As the gravitino $\tilde{G}$ being the lightest supersymmetric particle, the lightest neutralino $\tilde{\chi}_{1}^{0}$ decays into $\gamma \tilde{G}$. The production of $\tilde{\chi}_{1}^{0}$ in association with the light chargino $\tilde{\chi}_{1}^{ \pm}$will yield mono- $W \gamma$ signal. The current 95\% C.L. upper limit on the production cross section is of the order of $\mathcal{O}(10) \mathrm{fb}$ [91]. We expect higher discovery potential in the future. We also note that this mode has not been studied in the framework of the IDM.

Based on these discussions, we conclude that the mono- $W \gamma$ mode is one of the most sensitive channels to probe the $\operatorname{IDM}-U(1)$ at the LHC.

\section{V. $W^{ \pm} \gamma E_{T}^{\text {miss }}$ FINAL STATES AT THE LHC}

In this section, we make a comprehensive analysis of the mono- $W \gamma$ mode with the hadronic $W^{ \pm}$decay at the HLLHC and a future FCC-hh $100 \mathrm{TeV}$ collider:

$$
p p \rightarrow W^{ \pm}\left(\rightarrow q \bar{q}^{\prime}\right) \gamma+E_{T}^{\mathrm{miss}}
$$

From the comprehensive study of the theoretical and experimental constraints on the model, we take the following benchmark:

$$
\begin{aligned}
M_{S} & =70 \mathrm{GeV}, \quad M_{H^{ \pm}}=155 \mathrm{GeV}, \\
\lambda_{L} & =0.01, \quad \lambda_{2}=0.5 .
\end{aligned}
$$

The choice of $\lambda_{L}$ and $\lambda_{2}$ does not affect the mono- $W \gamma$ process. The parton-level cross section of the signal process is $\sigma \times \mathcal{B}\left(W^{ \pm} \rightarrow q \bar{q}^{\prime}\right)=3.23 \mathrm{fb} \quad(29.4 \mathrm{fb})$ at $\sqrt{s}=14 \mathrm{TeV}(100 \mathrm{TeV})$. The final state consists of a hard isolated photon, at least two jets, and large missing transverse momentum. For this final state, the backgrounds contaminating the searches fall into three categories:

(1) Irreducible backgrounds:
(a) $Z(\rightarrow \bar{\nu} \nu) \gamma+$ jets;
(b) $Z(\rightarrow \bar{\nu} \nu) Z(\rightarrow \bar{q} q) \gamma$;
(c) $W^{ \pm}\left(\rightarrow \bar{q} q^{\prime}\right) Z(\rightarrow \bar{\nu} \nu) \gamma$.

The first background of $Z \gamma+$ jets is dominant with the total cross section of $\sim 17(83) \mathrm{pb}$ at the 14 (100) TeV LHC while the other two are subleading.

(2) Reducible backgrounds involving the leptonic decay of a $W^{ \pm}$boson with the charged lepton escaping the detection (called $\ell_{\mathrm{esc}}^{ \pm}$). We consider three reducible backgrounds:
(a) $W^{ \pm}\left(\rightarrow \ell_{\mathrm{esc}}^{ \pm} \nu\right) \gamma+$ jets;
(b) $W^{ \pm}\left(\rightarrow \ell_{\mathrm{esc}}^{ \pm} \nu\right) W^{\mp}\left(\rightarrow \bar{q} q^{\prime}\right) \gamma$ and $W^{ \pm}\left(\rightarrow \ell_{\mathrm{esc}}^{ \pm} \nu\right) \times$ $Z(\rightarrow q \bar{q}) \gamma$
(c) $t \bar{t} \gamma$, followed by the semileptonic decay of the $t \bar{t}$ pair.

(3) Backgrounds from the fake photons. There exist nonzero probabilities of misidentifying an electron or a jet as a photon. The photon fake rates are usually taken as $P_{j \rightarrow \gamma}=5 \times 10^{-4}$ and $P_{e^{-} \rightarrow \gamma}=2 \%(5 \%)$ in the barrel (endcap) region, according to the combined study on the perspectives for the HL-LHC by the ATLAS and CMS collaborations [92]. However, they depend sensitively on the type of the process as well as the signal region. For example, the experimental study on the process $p p \rightarrow \gamma E_{T}^{\text {miss }}$ in the signal region with $E_{T}^{\text {miss }}>150 \mathrm{GeV}$ yields $P_{e^{-} \rightarrow \gamma}=$ $1.5 \%$ [88]. Since this type of background cannot be modeled in our analysis setup, especially at the $100 \mathrm{TeV}$ LHC, we will ignore the subleading backgrounds from the fake photons.

Signal and background processes are simulated at LO, using MadGraph5_aMC@NLO [81,82] with the NNPDF31 PDF set [93] and $\alpha_{s}\left(M_{Z}\right)=0.118$. For the renormalization and factorization scales, we choose

$$
\mu_{F, R}=\frac{1}{2} \sum_{i} \sqrt{p_{T, i}^{2}+m_{i}^{2}} .
$$

For $W^{ \pm} \gamma+$ jets and $\mathrm{Z} \gamma+$ jets, we simulated the productions with jet multiplicity up to two jets in the final state and merged them according to the MLM merging scheme [94] with a merging scale $Q_{0}=22.5 \mathrm{GeV}$. We have confirmed the stability of the calculations with respect to the variation of the merging scale.

In all of the simulations, we have generated events with some generator-level cuts on the photon transverse momentum $p_{T}^{\gamma}>5-10 \mathrm{GeV}$. The decays of $W, Z$, and the top quark were performed by using MadSpin [95]. PYTHIA8 was used for the showering and hadronization stages [96]. To include detector angularity and momentum smearing to the particle-level events, we used DELPHES as a fast-detector simulation tool [97] with the templates specific for the HLLHC and the FCC-hh. In the analysis, we cluster jets according to the anti- $k_{T}$ algorithm [98] with jet radius $D=$ 0.4 using energy flow as input. The clustering of jets was performed by FastJet [99].

After generating events to be called the "initial events," we take the five basic selection steps. First we demand $n_{\gamma} \geq 1$ and $n_{j} \geq 2$, i.e., at least one photon with $p_{T}^{\gamma}>$ $25 \mathrm{GeV}$ and $\left|\eta^{\gamma}\right|<2.47$, and at least two jets with $p_{T}^{j}>$ $25 \mathrm{GeV}$ and $\left|\eta^{j}\right|<2.5$. The second step is the lepton veto: we remove the event which contains at least one isolated lepton (electron or muon) with $p_{T}^{\ell}>7 \mathrm{GeV}$ and $\left|\eta^{\ell}\right|<2.5$. The third one is the $b$-jet veto, removing the event if the leading or subleading jet is $b$-tagged. The fourth and fifth steps are designed to reduce the $W^{ \pm}\left(\rightarrow \ell_{\mathrm{esc}}^{ \pm} \nu\right) \gamma+$ jets and $Z(\rightarrow \bar{\nu} \nu) \gamma+$ jets. We select events that contain one dijet candidate consistent with a hadronic decay of $W^{ \pm}$by demanding two jets to satisfy $\Delta R_{j_{1} j_{2}}<1$. If more than two pairs of jets are satisfying this condition, we keep the pair 
TABLE I. The number of events for the signal and the backgrounds after each of the five basic selection steps at the $14 \mathrm{TeV}$ LHC with the total integrated luminosity $\mathcal{L}=3 \mathrm{ab}^{-1}$. The background $V \gamma+$ jets refers to the combination of $Z(\rightarrow \bar{\nu} \nu) \gamma+$ jets and $W^{ \pm}\left(\rightarrow \ell_{\mathrm{esc}}^{ \pm} \nu\right) \gamma+$ jets, where $\ell_{\mathrm{esc}}^{ \pm}$denotes a charged lepton escaping the detection. The background $V V \gamma$ includes $Z(\rightarrow \bar{\nu} \nu) Z(\rightarrow \bar{q} q) \gamma, W^{ \pm}\left(\rightarrow \bar{q} q^{\prime}\right) Z(\rightarrow \bar{\nu} \nu) \gamma, W^{ \pm}\left(\rightarrow \ell_{\mathrm{esc}}^{ \pm} \nu\right) W^{\mp}\left(\rightarrow \bar{q} q^{\prime}\right) \gamma$, and $W^{ \pm}\left(\rightarrow \ell_{\mathrm{esc}}^{ \pm} \nu\right) Z(\rightarrow \bar{q} q) \gamma$.

\begin{tabular}{lccccc}
\hline \hline Selection & Signal & $V \gamma+$ jets & $t \bar{t} \gamma$ & $V V \gamma$ & $n_{s} / \sqrt{n_{b}}$ \\
\hline Initial events & $9.69 \times 10^{3}$ & $2.04 \times 10^{8}$ & $2.14 \times 10^{6}$ & $2.56 \times 10^{6}$ & $2.54 \times 10^{-3}$ \\
$n_{\gamma} \geq 1, n_{j} \geq 2$ & $1.90 \times 10^{3}$ & $1.40 \times 10^{7}$ & $7.60 \times 10^{5}$ & $4.48 \times 10^{5}$ & $6.83 \times 10^{-3}$ \\
Lepton veto & $1.89 \times 10^{3}$ & $9.38 \times 10^{6}$ & $4.02 \times 10^{5}$ & $3.72 \times 10^{5}$ & $1.02 \times 10^{-2}$ \\
$b$-tag veto & $1.77 \times 10^{3}$ & $8.94 \times 10^{6}$ & $1.40 \times 10^{5}$ & $3.30 \times 10^{5}$ & $1.03 \times 10^{-2}$ \\
$\Delta R_{j_{1} j_{2}}<1$ & $5.85 \times 10^{2}$ & $1.99 \times 10^{6}$ & $8.16 \times 10^{5}$ & $1.01 \times 10^{5}$ & $1.48 \times 10^{-2}$ \\
$\left|M_{j_{1} j_{2}}-m_{W}\right|<10 \mathrm{GeV}$ & $1.49 \times 10^{2}$ & $1.08 \times 10^{5}$ & $1.30 \times 10^{4}$ & $2.21 \times 10^{4}$ & $5.71 \times 10^{-2}$ \\
\hline \hline
\end{tabular}

TABLE II. Same as Table I but for the FCC-hh at $\sqrt{s}=100 \mathrm{TeV}$ and $\mathcal{L}=30 \mathrm{ab}^{-1}$.

\begin{tabular}{lccccc}
\hline \hline Selection & Signal & $V \gamma+$ jets & $t \bar{t} \gamma$ & $V V \gamma$ & $n_{s} / \sqrt{n_{b}}$ \\
\hline Initial events & $8.82 \times 10^{5}$ & $1.23 \times 10^{10}$ & $8.70 \times 10^{8}$ & $1.76 \times 10^{8}$ & $1.15 \times 10^{-2}$ \\
$n_{\gamma} \geq 1, n_{j} \geq 2$ & $1.71 \times 10^{5}$ & $1.46 \times 10^{9}$ & $3.36 \times 10^{8}$ & $3.13 \times 10^{7}$ & $1.62 \times 10^{-2}$ \\
Lepton veto & $1.71 \times 10^{5}$ & $8.73 \times 10^{8}$ & $1.67 \times 10^{8}$ & $2.55 \times 10^{7}$ & $2.78 \times 10^{-2}$ \\
$b$-tag veto & $1.64 \times 10^{5}$ & $8.33 \times 10^{8}$ & $5.65 \times 10^{7}$ & $2.30 \times 10^{7}$ & $3.11 \times 10^{-2}$ \\
$\Delta R_{j_{1} j_{2}}<1$ & $6.23 \times 10^{4}$ & $2.09 \times 10^{8}$ & $3.38 \times 10^{7}$ & $7.97 \times 10^{6}$ & $4.31 \times 10^{-2}$ \\
$\left|M_{j_{1} j_{2}}-m_{W}\right|<10 \mathrm{GeV}$ & $1.86 \times 10^{4}$ & $1.66 \times 10^{7}$ & $6.64 \times 10^{6}$ & $2.02 \times 10^{6}$ & $1.28 \times 10^{-1}$ \\
\hline \hline
\end{tabular}

with the minimum $\Delta R_{j_{1} j_{2}}$. The dijet with $\Delta R_{j_{1} j_{2}}<1$ is further required to satisfy $\left|M_{j_{1} j_{2}}-m_{W}\right|<10 \mathrm{GeV}$ with $m_{W}=80.4 \mathrm{GeV}$.

In Tables I and II, we show the cut-flows for the signal and the backgrounds at the $14 \mathrm{TeV}$ and $100 \mathrm{TeV}$ LHC, respectively. In order to assess the discovery potential of the signal process, we present the signal significance at each selection step, defined by [100]

$$
\mathcal{S}=\frac{n_{s}}{\sqrt{n_{b}}}
$$

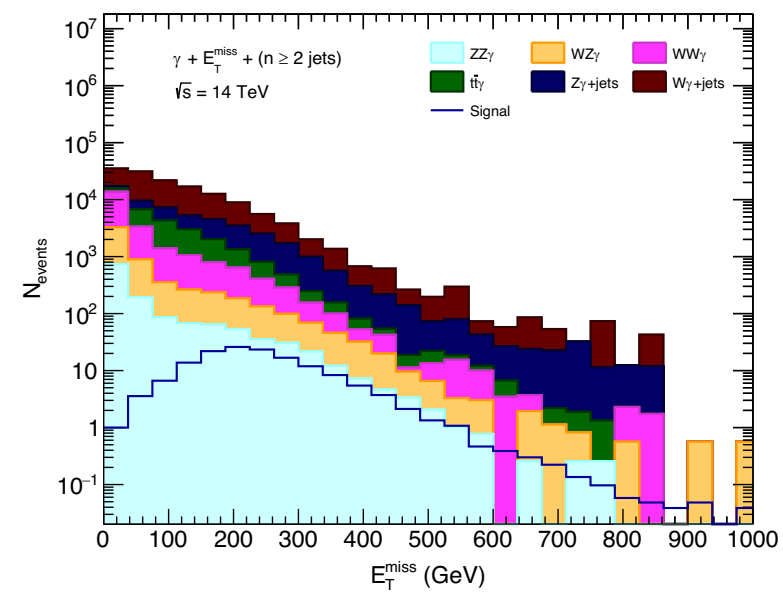

where $n_{s}$ and $n_{b}$ are the number of events for the signal and backgrounds, respectively. Among the basic selection steps, the $W$-boson mass requirement significantly reduces the expected number of background events. However, the backgrounds are still overwhelming, yielding the significance of the order of $10^{-2}\left(10^{-1}\right)$ at the $14 \mathrm{TeV}(100 \mathrm{TeV})$. Therefore, we need to devise a new method in order to enhance the significance.

In Figs. 5 and 6, we present the distributions of the missing transverse energy, $E_{T}^{\text {miss }}$, and the scalar sum of the transverse momenta of jets, $H_{T}$, at the HL-LHC (left panel)

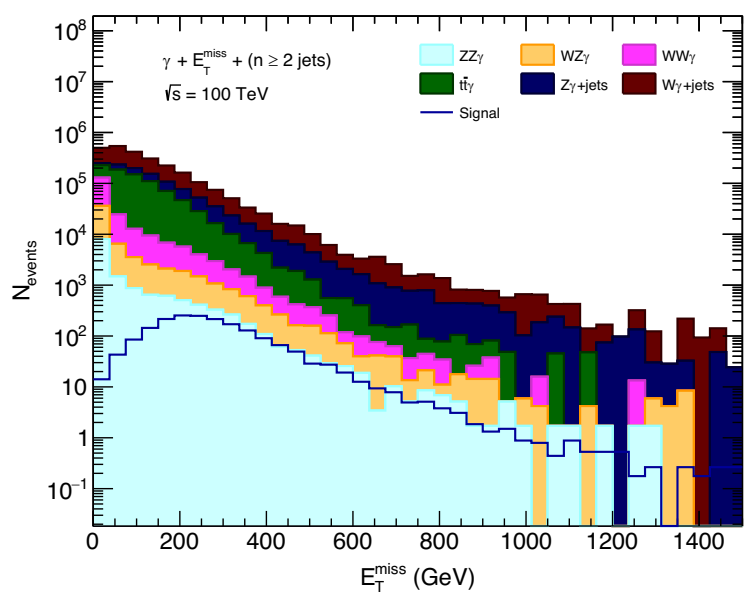

FIG. 5. Expected number of events for the signal (blue line) and the backgrounds stacked on the top of each other as a function of the missing transverse energy at the HL-LHC (left panel) and the FCC-hh (right panel). The distributions are shown after the basic selections. 

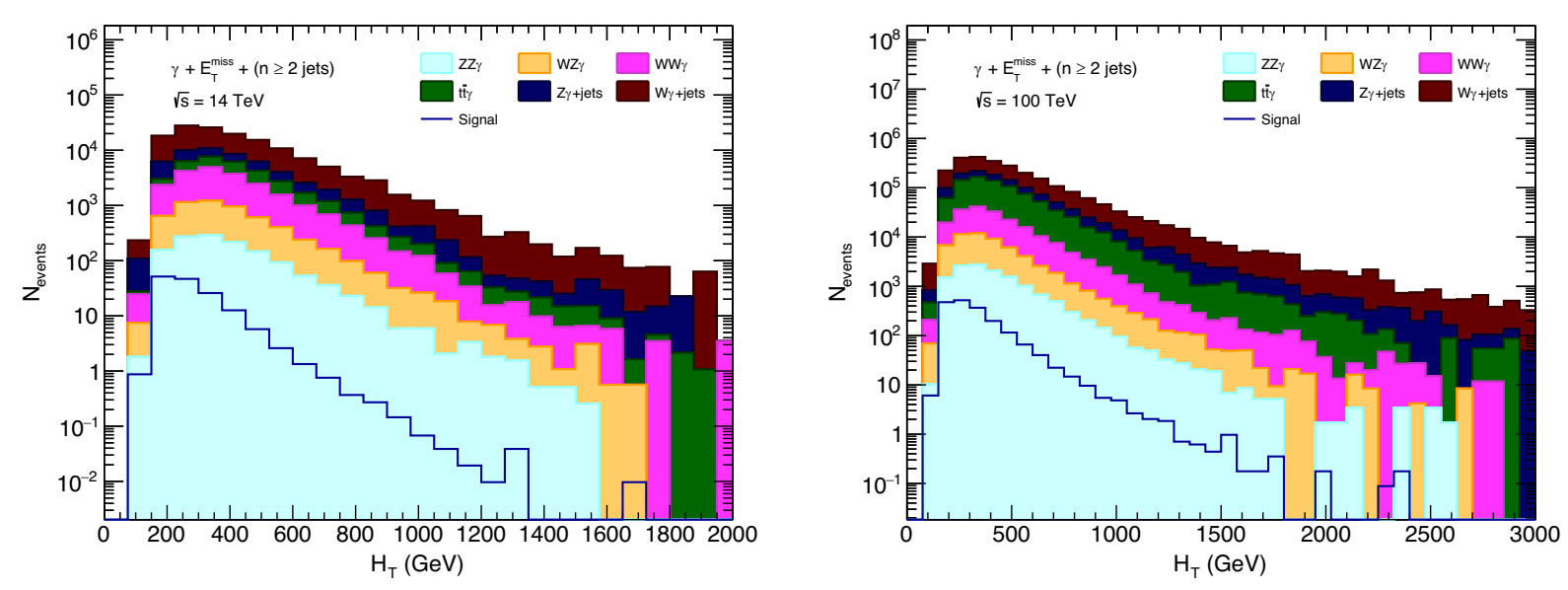

FIG. 6. Same as Fig. 5 but for the scalar sum of the transverse momenta of jets.

and the FCC-hh (right panel). Unfortunately, the $E_{T}^{\text {miss }}$ distributions of the signal have a similar distribution shape to those of the backgrounds. Requiring large missing transverse energy does not improve the signal-to-background ratios. This is attributed to the light DM mass, $M_{S}=70 \mathrm{GeV}$, which is inevitable for the condition $\Omega_{h_{1,2}} / \Omega_{\mathrm{DM}}^{\text {Planck }}>0.1$ to be satisfied. The $H_{T}$ distribution shows slight differences between the signal and the backgrounds such that the backgrounds have stronger $H_{T}$ than the signal. Based on these characteristics, we suggest the use of the modified $E_{T}^{\text {miss }}$ significance, $\mathcal{P}_{E_{T}^{\text {miss }}}$, defined by

$$
\mathcal{P}_{E_{T}^{\text {miss }}}=\frac{E_{T}^{\text {miss }}}{\sqrt{H_{T}}}
$$

The event yields for $\mathcal{P}_{E_{T}^{\text {miss }}}$ at the HL-LHC and the FCC-hh are shown in Fig. 7. We can see that the signal has a peak around $\mathcal{P}_{E_{T}^{\text {miss }}} \simeq 15 \sqrt{\mathrm{GeV}}$ while all of the backgrounds

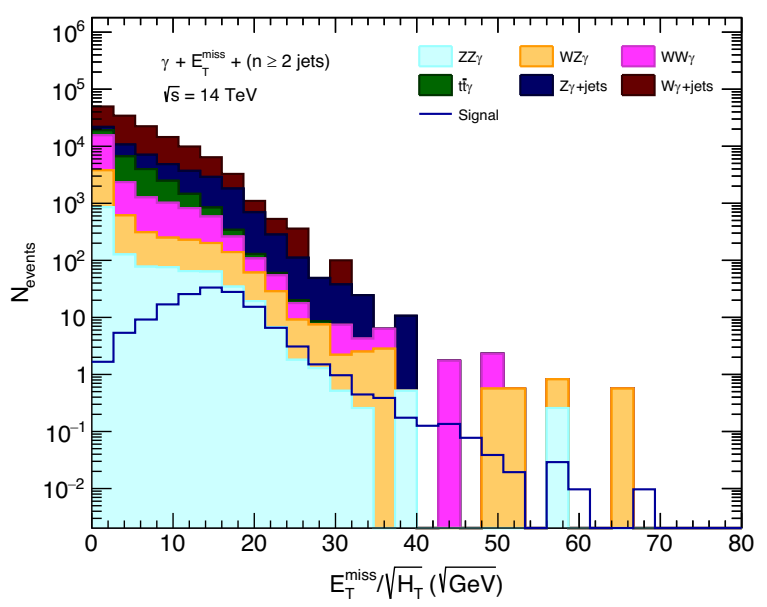

have monotonically decreasing shape. Therefore, selecting $\mathcal{P}_{E_{T}^{\text {miss }}}$ larger than a certain value, called $\mathcal{C}_{\text {min }}$, could help to improve the signal significance.

In order to find the optimized cut on $\mathcal{P}_{E_{T}^{\text {miss }}}$, we present the significance as a function of $\mathcal{C}_{\min }$ for the HL-LHC (left panel) and for the FCC-hh at $100 \mathrm{TeV}$ (right panel) in Fig. 8. We consider two cases of $E_{T}^{\text {miss }}>50 \mathrm{GeV}$ (green line) and $E_{T}^{\text {miss }}>250 \mathrm{GeV}$ (brown line). Both at the HLLHC and FCC-hh, the cut of $E_{T}^{\text {miss }}>50 \mathrm{GeV}$ yields higher significance than the cut of $E_{T}^{\text {miss }}>250 \mathrm{GeV}$ for $\mathcal{C}_{\text {min }} \lesssim 18 \mathrm{GeV}$. Therefore, the optimal cuts on $E_{T}^{\text {miss }}$ and $\mathcal{P}_{E_{T}^{\text {miss }}}$ are

$$
E_{T}^{\text {miss }} \geq 50 \mathrm{GeV}, \quad \mathcal{P}_{E_{T}^{\text {miss }}} \geq 15 \sqrt{\mathrm{GeV}}
$$

Then the signal significance at the LH-LHC is about 0.77 , leading to a disappointing result that the IDM with $U(1)$ symmetry cannot be probed at the HL-LHC. At the FCC-hh with $\sqrt{s}=100 \mathrm{TeV}$ and $30 \mathrm{ab}^{-1}$, the significance can be as

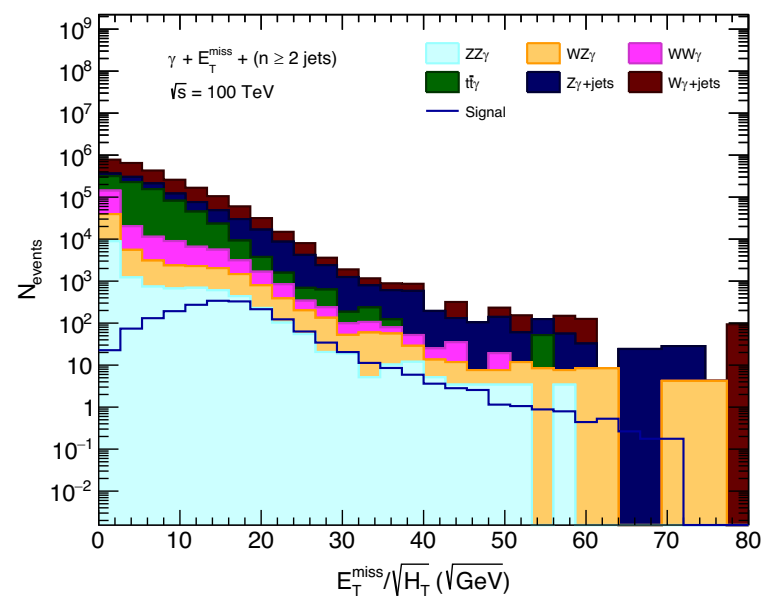

FIG. 7. Same as Fig. 5 but for the modified $E_{T}^{\text {miss }}$ significance $E_{T}^{\text {miss }} / \sqrt{H_{T}}$. 

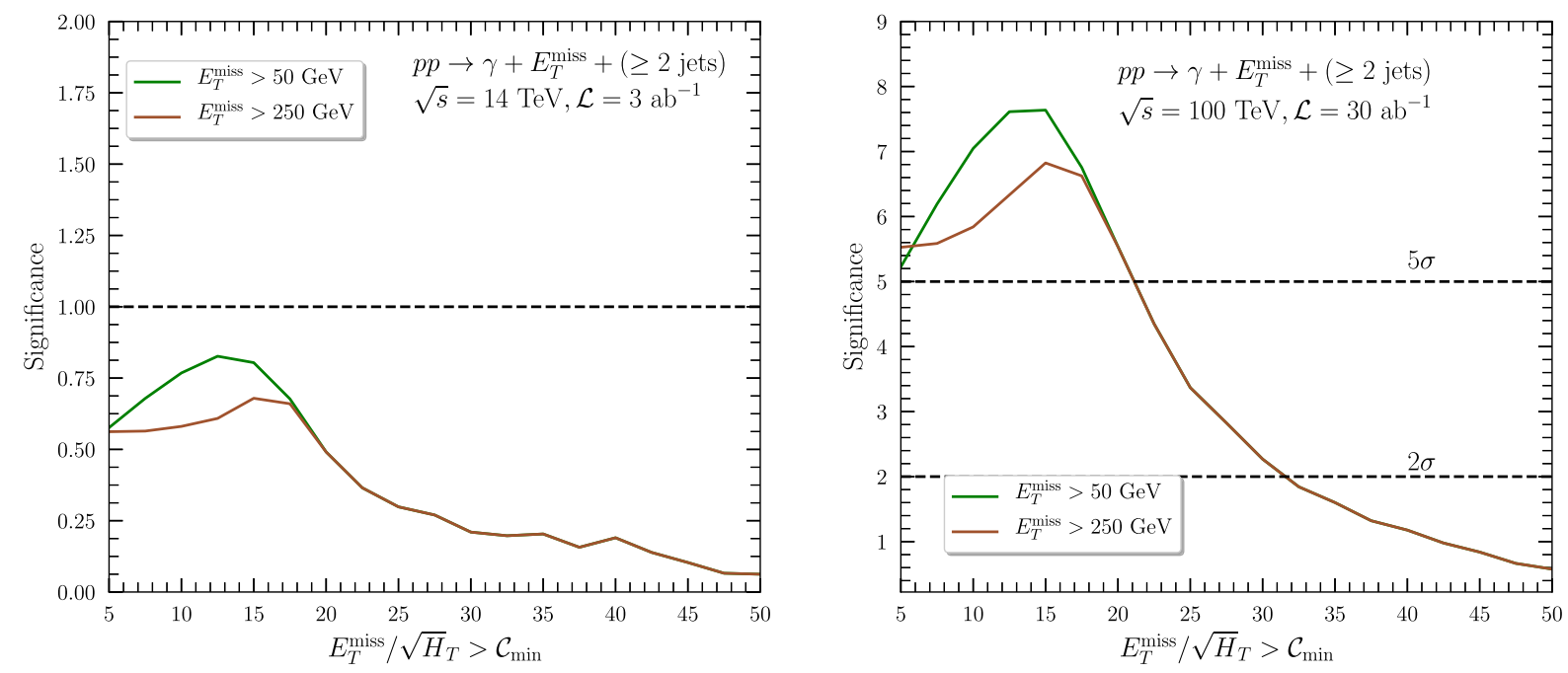

FIG. 8. Signal significance as a function of the cut on the modified $E_{T}^{\text {miss }}$ significance $\mathcal{P}_{E_{T}^{\text {miss }}}$ at the HL-LHC and the FCC-hh (right panel).

high as about 7.5. The IDM- $U(1)$ has a chance to be probed through the mono- $W \gamma$ mode.

\section{CONCLUSIONS}

We have comprehensively studied the phenomenology of the inert doublet model (IDM) with a global $U(1)$ symmetry. The model has an additional Higgs doublet field $\Phi^{\prime}$, accommodating new scalar bosons, $h_{1}, h_{2}$, and $H^{ \pm}$. By promoting the usually adopted $Z_{2}$ parity into a global $U(1)$ symmetry, the theory provides two DM particles, neutral $C P$-even and $C P$-odd scalar bosons, $h_{1}$ and $h_{2}$. The extended symmetry strongly constrains the model. As the $\lambda_{5}$ term in the scalar potential is prohibited by the $U(1)$ symmetry, two important results occur. First, $h_{1}$ and $h_{2}$ have the same mass and thus become DM candidates. Second, the number of model parameters is one less than that in the IDM with $Z_{2}$ parity. The theory becomes very limited by the combination of various constraints such as the LEP experiments, electroweak oblique parameters, theoretical stability, Higgs precision data, DM relic density, and DM direct detection experiments. Particularly when we demand that the IDM- $U(1)$ explains at least $10 \%$ of the observed DM relic density, the parameter space that survived is very narrow: the DM mass $M_{S}$ is about $70 \mathrm{GeV}$; the parameter $\lambda_{L}$, which governs the couplings of $h_{1}$ and $h_{2}$ to the SM-like Higgs boson $H$, is extremely small; the charged Higgs boson cannot be heavier than about $M_{S}+100 \mathrm{GeV}$.

For the LHC phenomenology of the model, we first studied the production cross sections of the inert scalar bosons in the mono- $X$ and mono- $X X^{\prime}$ channels. The key factor is the mass difference between the charged Higgs boson and the DM particles, $\Delta M$. If $\Delta M$ is above the $W$ boson mass so that the charged Higgs boson decays into $W^{ \pm} h_{1,2}$ on-shell, the production cross sections involving $W^{ \pm}$, mono- $W$, mono- $W Z$, mono- $W W$, and mono- $W W$, are highly enhanced, compared with the case of $\Delta M<m_{W}$. Nevertheless, the cross sections are still small. Based on the projection of the current experimental results of the $13 \mathrm{TeV}$ LHC into the HLLHC, we expected that the mono- $W \gamma$ process has a high chance to probe the model.

Focusing on the hadronic decay mode of the $W^{ \pm}$boson, we have completed the analysis with full detector-level simulations for the mono- $W \gamma$ signal. Partially because the DM mass is rather light like $\sim 70 \mathrm{GeV}$ in this model, the kinematical distributions of the transverse momentum of the leading-isolated photon and the missing transverse energy are similar for both the signal and the backgrounds. We found that the scalar sum of the transverse momenta of jets, $H_{T}$, for the signal is softer than that for the backgrounds. So we suggested the use of the modified $E_{T}^{\text {miss }}$ significance, $\mathcal{P}_{E_{T}^{\text {miss }}}=E_{T}^{\text {miss }} / \sqrt{H_{T}}$, as a key observable to reduce the backgrounds: requiring $\mathcal{P}_{E_{T}^{\text {miss }}}>\mathcal{C}_{\text {min }}$ is shown to be very efficient. Although the cut on $\mathcal{P}_{E_{T}^{\text {miss }}}$ enhances the signal significance about tenfold, the maximum significance at the $14 \mathrm{TeV}$ LHC with the total integrated luminosity of $3 \mathrm{ab}^{-1}$ is below one. It is not feasible to probe the model at the HL-LHC. Extending the analysis into the future FCC-hh at $\sqrt{s}=100 \mathrm{TeV}$ with the total integrated luminosity of $30 \mathrm{ab}^{-1}$, the signal significance is shown to reach about 7.5. The IDM-U(1) has a chance to be probed through the mono- $W \gamma$ mode.

\section{ACKNOWLEDGMENTS}

This work is supported by the National Research Foundation of Korea, Grant No. NRF2019R1A2C1009419. The work of S. S. is supported by IBS under the project code, IBS-R018-D1. 
[1] G. Bertone, D. Hooper, and J. Silk, Particle dark matter: Evidence, candidates and constraints, Phys. Rep. 405, 279 (2005).

[2] Q.-H. Cao, C.-R. Chen, C. S. Li, and H. Zhang, Effective dark matter model: Relic density, CDMS II, Fermi LAT and LHC, J. High Energy Phys. 08 (2011) 018.

[3] M. Beltrán, D. Hooper, E. W. Kolb, Z. A. Krusberg, and T. M. Tait, Maverick dark matter at colliders, J. High Energy Phys. 09 (2010) 037.

[4] Y. Bai, P. J. Fox, and R. Harnik, The tevatron at the frontier of dark matter direct detection, J. High Energy Phys. 12 (2010) 048.

[5] J. Goodman, M. Ibe, A. Rajaraman, W. Shepherd, T. M. Tait, and H.-B. Yu, Gamma ray line constraints on effective theories of dark matter, Nucl. Phys. B844, 55 (2011).

[6] P. J. Fox, R. Harnik, J. Kopp, and Y. Tsai, Missing energy signatures of dark matter at the LHC, Phys. Rev. D 85, 056011 (2012).

[7] G. Busoni, A. De Simone, E. Morgante, and A. Riotto, On the validity of the effective field theory for dark matter searches at the LHC, Phys. Lett. B 728, 412 (2014).

[8] A. Beniwal, F. Rajec, C. Savage, P. Scott, C. Weniger, M. White, and A. G. Williams, Combined analysis of effective Higgs portal dark matter models, Phys. Rev. D 93, 115016 (2016).

[9] F. Bishara, J. Brod, B. Grinstein, and J. Zupan, Chiral effective theory of dark matter direct detection, J. Cosmol. Astropart. Phys. 02 (2017) 009.

[10] H. An, X. Ji, and L.-T. Wang, Light dark matter and $Z^{\prime}$ dark force at colliders, J. High Energy Phys. 07 (2012) 182.

[11] M. T. Frandsen, F. Kahlhoefer, A. Preston, S. Sarkar, and K. Schmidt-Hoberg, LHC and tevatron bounds on the dark matter direct detection cross-section for vector mediators, J. High Energy Phys. 07 (2012) 123.

[12] H. Dreiner, D. Schmeier, and J. Tattersall, Contact interactions probe effective dark matter models at the LHC, Europhys. Lett. 102, 51001 (2013).

[13] J. Abdallah et al., Simplified models for dark matter searches at the LHC, Phys. Dark Universe 9-10, 8 (2015).

[14] A. Alves, A. Berlin, S. Profumo, and F. S. Queiroz, Diracfermionic dark matter in $\mathrm{U}(1)_{X}$ models, J. High Energy Phys. 10 (2015) 076.

[15] A. Alves, A. Berlin, S. Profumo, and F. S. Queiroz, Dark matter complementarity and the $Z^{\prime}$ portal, Phys. Rev. D 92, 083004 (2015).

[16] A. Alves, D. A. Camargo, A. G. Dias, R. Longas, C. C. Nishi, and F. S. Queiroz, Collider and dark matter searches in the inert doublet model from Peccei-Quinn symmetry, J. High Energy Phys. 10 (2016) 015.

[17] G. Busoni et al., Recommendations on presenting LHC searches for missing transverse energy signals using simplified $s$-channel models of dark matter, Phys. Dark Universe 27, 100365 (2020).

[18] F. Kahlhoefer, K. Schmidt-Hoberg, T. Schwetz, and S. Vogl, Implications of unitarity and gauge invariance for simplified dark matter models, J. High Energy Phys. 02 (2016) 016.

[19] M. Bauer, U. Haisch, and F. Kahlhoefer, Simplified dark matter models with two Higgs doublets: I. Pseudoscalar mediators, J. High Energy Phys. 05 (2017) 138.
[20] A. De Simone and T. Jacques, Simplified models vs. effective field theory approaches in dark matter searches, Eur. Phys. J. C 76, 367 (2016).

[21] N. G. Deshpande and E. Ma, Pattern of symmetry breaking with two Higgs doublets, Phys. Rev. D 18, 2574 (1978).

[22] T. A. Chowdhury, M. Nemevsek, G. Senjanović, and Y. Zhang, Dark matter as the trigger of strong electroweak phase transition, J. Cosmol. Astropart. Phys. 02 (2012) 029.

[23] D. Borah and J. M. Cline, Inert doublet dark matter with strong electroweak phase transition, Phys. Rev. D 86, 055001 (2012).

[24] N. Blinov, S. Profumo, and T. Stefaniak, The electroweak phase transition in the inert doublet model, J. Cosmol. Astropart. Phys. 07 (2015) 028.

[25] J. M. Cline and K. Kainulainen, Improved electroweak phase transition with subdominant inert doublet dark matter, Phys. Rev. D 87, 071701 (2013).

[26] E. Ma, Verifiable radiative seesaw mechanism of neutrino mass and dark matter, Phys. Rev. D 73, 077301 (2006).

[27] L. L. Honorez, E. Nezri, J. F. Oliver, and M. H. Tytgat, The inert doublet model: An archetype for dark matter, J. Cosmol. Astropart. Phys. 02 (2007) 028.

[28] T. Hambye, F.-S. Ling, L. L. Honorez, and J. Rocher, Scalar multiplet dark matter, J. High Energy Phys. 07 (2009) 090.

[29] L. L. Honorez and C. E. Yaguna, A new viable region of the inert doublet model, J. Cosmol. Astropart. Phys. 01 (2011) 002.

[30] A. Goudelis, B. Herrmann, and O. Stål, Dark matter in the inert doublet model after the discovery of a Higgs-like boson at the LHC, J. High Energy Phys. 09 (2013) 106.

[31] E. M. Dolle and S. Su, The inert dark matter, Phys. Rev. D 80, 055012 (2009).

[32] M. Gustafsson, E. Lundström, L. Bergström, and J. Edsjö, Significant Gamma Lines from Inert Higgs Dark Matter, Phys. Rev. Lett. 99, 041301 (2007).

[33] Q.-H. Cao, E. Ma, and G. Rajasekaran, Observing the dark scalar doublet and its impact on the standard-model Higgs boson at colliders, Phys. Rev. D 76, 095011 (2007).

[34] P. Agrawal, E. M. Dolle, and C. A. Krenke, Signals of inert doublet dark matter in neutrino telescopes, Phys. Rev. D 79, 015015 (2009).

[35] S. Andreas, M. H. Tytgat, and Q. Swillens, Neutrinos from inert doublet dark matter, J. Cosmol. Astropart. Phys. 04 (2009) 004.

[36] E. Dolle, X. Miao, S. Su, and B. Thomas, Dilepton signals in the inert doublet model, Phys. Rev. D 81, 035003 (2010).

[37] E. Nezri, M. H. Tytgat, and G. Vertongen, $e^{+}$and $\bar{p}$ from inert doublet model dark matter, J. Cosmol. Astropart. Phys. 04 (2009) 014.

[38] X. Miao, S. Su, and B. Thomas, Trilepton signals in the inert doublet model, Phys. Rev. D 82, 035009 (2010).

[39] M. Gustafsson, S. Rydbeck, L. Lopez-Honorez, and E. Lundström, Status of the inert doublet model and the role of multileptons at the LHC, Phys. Rev. D 86, 075019 (2012).

[40] A. Arhrib, R. Benbrik, and N. Gaur, $H \rightarrow \gamma \gamma$ in inert Higgs doublet model, Phys. Rev. D 85, 095021 (2012). 
[41] B. Świeżewska and M. Krawczyk, Diphoton rate in the inert doublet model with a $125 \mathrm{GeV}$ Higgs boson, Phys. Rev. D 88, 035019 (2013).

[42] M. Krawczyk, D. Sokołowska, P. Swaczyna, and B. Świeżewska, Constraining inert dark matter by $R_{\gamma y}$ and WMAP data, J. High Energy Phys. 09 (2013) 055.

[43] M. Aoki, S. Kanemura, and H. Yokoya, Reconstruction of inert doublet scalars at the international linear collider, Phys. Lett. B 725, 302 (2013).

[44] C. Garcia-Cely and A. Ibarra, Novel Gamma-ray spectral features in the inert doublet model, J. Cosmol. Astropart. Phys. 09 (2013) 025.

[45] A. Arhrib, Y.-L. S. Tsai, Q. Yuan, and T.-C. Yuan, An updated analysis of inert Higgs doublet model in light of the recent results from LUX, PLANCK, AMS-02 and LHC, J. Cosmol. Astropart. Phys. 06 (2014) 030.

[46] A. J. Ilnicka, M. Krawczyk, and T. Robens, Inert doublet model in the light of LHC and astrophysical data, Proc. Sci., EPS-HEP2015 (2015) 143 [arXiv:1510.04159].

[47] G. Bélanger, B. Dumont, A. Goudelis, B. Herrmann, S. Kraml, and D. Sengupta, Dilepton constraints in the inert doublet model from Run 1 of the LHC, Phys. Rev. D 91, 115011 (2015).

[48] A. Arhrib, R. Benbrik, J. El Falaki, and A. Jueid, Radiative corrections to the triple Higgs coupling in the inert Higgs doublet model, J. High Energy Phys. 12 (2015) 007.

[49] A. Ilnicka, M. Krawczyk, and T. Robens, Inert doublet model in light of LHC Run I and astrophysical data, Phys. Rev. D 93, 055026 (2016).

[50] A. Belyaev, G. Cacciapaglia, I. P. Ivanov, F. Rojas-Abatte, and M. Thomas, Anatomy of the inert two Higgs doublet model in the light of the LHC and non-LHC dark matter searches, Phys. Rev. D 97, 035011 (2018).

[51] P. Poulose, S. Sahoo, and K. Sridhar, Exploring the inert doublet model through the dijet plus missing transverse energy channel at the LHC, Phys. Lett. B 765, 300 (2017).

[52] S. Kanemura, M. Kikuchi, and K. Sakurai, Testing the dark matter scenario in the inert doublet model by future precision measurements of the Higgs boson couplings, Phys. Rev. D 94, 115011 (2016).

[53] S. Banerjee and N. Chakrabarty, A revisit to scalar dark matter with radiative corrections, J. High Energy Phys. 05 (2019) 150.

[54] A. Belyaev, S. Moretti, T. R. Fernandez Perez Tomei, S. F. Novaes, P. G. Mercadante, C. S. Moon, L. Panizzi, F. Rojas, and M. Thomas, Advancing LHC probes of dark matter from the inert two-Higgs-doublet model with the monojet signal, Phys. Rev. D 99, 015011 (2019).

[55] J. Kalinowski, W. Kotlarski, T. Robens, D. Sokołowska, and A. F. Żarnecki, Exploring inert scalars at CLIC, J. High Energy Phys. 07 (2019) 053.

[56] S. Banerjee, F. Boudjema, N. Chakrabarty, G. Chalons, and H. Sun, Relic density of dark matter in the inert doublet model beyond leading order: The heavy mass case, Phys. Rev. D 100, 095024 (2019).

[57] A. Bhardwaj, P. Konar, T. Mandal, and S. Sadhukhan, Probing the inert doublet model using jet substructure with a multivariate analysis, Phys. Rev. D 100, 055040 (2019).
[58] P. Bandyopadhyay, P. B. Dev, S. Jangid, and A. Kumar, Vacuum stability in inert Higgs doublet model with righthanded neutrinos, J. High Energy Phys. 08 (2020) 154.

[59] Y. Guo-He, S. Mao, L. Gang, Z. Yu, and G. Jian-You, Searches for dark matter via charged Higgs pair production in the inert doublet model at $\gamma \gamma$ collider, arXiv:2006.06216.

[60] P. Ko, Y. Omura, and C. Yu, A resolution of the flavor problem of two Higgs doublet models with an extra U(1)_H symmetry for Higgs flavor, Phys. Lett. B 717, 202 (2012).

[61] P. Ko, Y. Omura, and C. Yu, Higgs phenomenology in Type-I $2 \mathrm{HDM}$ with $U(1)_{H}$ Higgs gauge symmetry, J. High Energy Phys. 01 (2014) 016.

[62] P. Ko, Y. Omura, and C. Yu, Dark matter and dark force in the type-I inert $2 \mathrm{HDM}$ with local $\mathrm{U}(1)_{H}$ gauge symmetry, J. High Energy Phys. 11 (2014) 054.

[63] P. Ko, Y. Omura, and C. Yu, Higgs and dark matter physics in the type-II two-Higgs-doublet model inspired by $E_{6}$ GUT, J. High Energy Phys. 06 (2015) 034.

[64] C. Arina, F.-S. Ling, and M. H. Tytgat, IDM and iDM or the inert doublet model and inelastic dark matter, J. Cosmol. Astropart. Phys. 10 (2009) 018.

[65] A. D. Plascencia, Classical scale invariance in the inert doublet model, J. High Energy Phys. 09 (2015) 026.

[66] H. E. Haber, O. M. Ogreid, P. Osland, and M. N. Rebelo, Symmetries and mass degeneracies in the scalar sector, J. High Energy Phys. 01 (2019) 042.

[67] G. Apollinari, O. Brüning, T. Nakamoto, and L. Rossi, High Luminosity Large Hadron Collider HL-LHC, CERN Yellow Rep. (2015), pp. 1-19, http://dx.doi.org/10.5170/ CERN-2015-005.1.

[68] A. Abada et al. (FCC Collaboration), FCC-hh: The hadron collider: Future circular collider conceptual design report volume 3, Eur. Phys. J. Special Topics 228, 755 (2019).

[69] D. Tucker-Smith and N. Weiner, Inelastic dark matter, Phys. Rev. D 64, 043502 (2001).

[70] M. Tanabashi et al. (Particle Data Group), Review of particle physics, Phys. Rev. D 98, 030001 (2018).

[71] A. Pierce and J. Thaler, Natural dark matter from an unnatural Higgs boson and new colored particles at the TeV scale, J. High Energy Phys. 08 (2007) 026.

[72] N. Blinov, J. Kozaczuk, D. E. Morrissey, and A. de la Puente, Compressing the inert doublet model, Phys. Rev. D 93, 035020 (2016).

[73] Y.-L. S. Tsai, V. Q. Tran, and C.-T. Lu, Confronting dark matter co-annihilation of inert two Higgs doublet model with a compressed mass spectrum, J. High Energy Phys. 06 (2020) 033.

[74] V. Khachatryan et al. (CMS collaboration), Search for disappearing tracks in proton-proton collisions at $\sqrt{s}=8 \mathrm{TeV}$, J. High Energy Phys. 01 (2015) 096.

[75] G. Aad et al. (ATLAS Collaboration), Combined measurements of Higgs boson production and decay using up to $80 \mathrm{fb}^{-1}$ of proton-proton collision data at $\sqrt{s}=13 \mathrm{TeV}$ collected with the ATLAS experiment, Phys. Rev. D 101, 012002 (2020).

[76] I. F. Ginzburg, K. A. Kanishev, M. Krawczyk, and D. Sokołowska, Evolution of Universe to the present inert phase, Phys. Rev. D 82, 123533 (2010). 
[77] A. Ahriche, A. Arhrib, A. Jueid, S. Nasri, and A. de La Puente, Mono-Higgs signature in the scotogenic model with Majorana dark matter, Phys. Rev. D 101, 035038 (2020).

[78] P. A. R. Ade et al. (Planck Collaboration), Planck 2015 results. XIII. Cosmological parameters, Astron. Astrophys. 594, A13 (2016).

[79] G. Bélanger, F. Boudjema, A. Goudelis, A. Pukhov, and B. Zaldivar, micromegas5.0: Freeze-in, Comput. Phys. Commun. 231, 173 (2018).

[80] E. Aprile et al. (XENON Collaboration), First Dark Matter Search Results from the XENON1T Experiment, Phys. Rev. Lett. 119, 181301 (2017).

[81] J. Alwall, M. Herquet, F. Maltoni, O. Mattelaer, and T. Stelzer, MadGraph_5: Going beyond, J. High Energy Phys. 06 (2011) 128.

[82] J. Alwall, R. Frederix, S. Frixione, V. Hirschi, F. Maltoni, O. Mattelaer, H.-S. Shao, T. Stelzer, P. Torrielli, and M. Zaro, The automated computation of tree-level and nextto-leading order differential cross sections, and their matching to parton shower simulations, J. High Energy Phys. 07 (2014) 079.

[83] C. Degrande, C. Duhr, B. Fuks, D. Grellscheid, O. Mattelaer, and T. Reiter, UFO-The universal FeynRules output, Comput. Phys. Commun. 183, 1201 (2012).

[84] B. Eiteneuer, A. Goudelis, and J. Heisig, The inert doublet model in the light of Fermi-LAT gamma-ray data: A global fit analysis, Eur. Phys. J. C 77, 624 (2017).

[85] M. Aaboud et al. (ATLAS Collaboration), Search for dark matter in events with a hadronically decaying vector boson and missing transverse momentum in $p p$ collisions at $\sqrt{s}=13 \mathrm{TeV}$ with the ATLAS detector, J. High Energy Phys. 10 (2018) 180.

[86] G. Aad et al. (ATLAS Collaboration), Measurement of $W^{ \pm}$ and Z-boson production cross sections in $p p$ collisions at $\sqrt{s}=13 \mathrm{TeV}$ with the ATLAS detector, Phys. Lett. B 759, 601 (2016).

[87] A. Ahriche, A. Jueid, and S. Nasri, Radiative neutrino mass and Majorana dark matter within an inert Higgs doublet model, Phys. Rev. D 97, 095012 (2018).

[88] M. Aaboud et al. (ATLAS Collaboration), Search for dark matter at $\sqrt{s}=13 \mathrm{TeV}$ in final states containing an energetic photon and large missing transverse momentum with the ATLAS detector, Eur. Phys. J. C 77, 393 (2017).

[89] A. M. Sirunyan et al. (CMS Collaboration), Search for new physics in final states with a single photon and missing transverse momentum in proton-proton collisions at $\sqrt{s}=13 \mathrm{TeV}, \mathrm{J}$. High Energy Phys. 02 (2019) 074.

[90] ATLAS Collaboration, Search for supersymmetry in events with at least one photon, one lepton, and large missing transverse momentum in proton-proton collision at a center-of-mass energy of $7 \mathrm{TeV}$ with the ATLAS detector, Report No. ATLAS-CONF-2012-144, https:// inspirehep.net/literature/1230007.

[91] A. M. Sirunyan et al. (CMS Collaboration), Search for supersymmetry in events with a photon, a lepton, and missing transverse momentum in proton-proton collisions at $\sqrt{s}=13 \mathrm{TeV}$, J. High Energy Phys. 01 (2019) 154.

[92] ATLAS and CMS Collaboration, Addendum to the Report on the Physics at the HL-LHC, and Perspectives for the HE-LHC: Collection of Notes from ATLAS and CMS, CERN Yellow Rep. Monogr. Vol. 7 (2019), https://doi.org/ 10.23731/CYRM-2019-007.Addendum.

[93] R. A. Khalek et al. (NNPDF Collaboration), Parton distributions with theory uncertainties: General formalism and first phenomenological studies, Eur. Phys. J. C 79, 931 (2019).

[94] M. L. Mangano, M. Moretti, F. Piccinini, and M. Treccani, Matching matrix elements and shower evolution for top-quark production in hadronic collisions, J. High Energy Phys. 01 (2007) 013.

[95] P. Artoisenet, R. Frederix, O. Mattelaer, and R. Rietkerk, Automatic spin-entangled decays of heavy resonances in Monte Carlo simulations, J. High Energy Phys. 03 (2013) 015 .

[96] T. Sjöstrand, S. Ask, J. R. Christiansen, R. Corke, N. Desai, P. Ilten, S. Mrenna, S. Prestel, C. O. Rasmussen, and P. Z. Skands, An introduction to PYTHIA8.2, Comput. Phys. Commun. 191, 159 (2015).

[97] J. de Favereau, C. Delaere, P. Demin, A. Giammanco, V. Lematre, A. Mertens, and M. Selvaggi (DELPHES 3 Collaboration), DELPHES 3, A modular framework for fast simulation of a generic collider experiment, J. High Energy Phys. 02 (2014) 057.

[98] M. Cacciari, G. P. Salam, and G. Soyez, The anti- $k_{t}$ jet clustering algorithm, J. High Energy Phys. 04 (2008) 063.

[99] M. Cacciari, G. P. Salam, and G. Soyez, FastJet user manual, Eur. Phys. J. C 72, 1896 (2012).

[100] G. Cowan, K. Cranmer, E. Gross, and O. Vitells, Asymptotic formulae for likelihood-based tests of new physics, Eur. Phys. J. C 71, 1554 (2011). 